\title{
Liquid crystal defects in the Landau-de Gennes theory in two dimensions - beyond the one-constant approximation
}

\author{
Georgy Kitavtsev*, Jonathan M Robbins*, \\ Valeriy Slastikov*, Arghir Zarnescu ${ }^{\dagger \ddagger \S}$
}

January 16, 2017

\begin{abstract}
We consider the two-dimensional Landau-de Gennes energy with several elastic constants, subject to general $k$-radially symmetric boundary conditions. We show that for generic elastic constants the critical points consistent with the symmetry of the boundary conditions exist only in the case $k=2$. In this case we identify three types of radial profiles: with two, three of full five components and numerically investigate their minimality and stability depending on suitable parameters.

We also numerically study the stability properties of the critical points of the Landau-de Gennes energy and capture the intricate dependence of various qualitative features of these solutions on the elastic constants and the physical regimes of the liquid crystal system.
\end{abstract}

\section{Introduction}

Nematic liquid crystals are amongst the simplest complex materials. They are liquid solutions whose microstructure is determined by the local orientational order of their rod-like solute particles. This microstructure is responsible for the anisotropic optical properties on which LCD technologies are based. Defects in nematic liquid crystals are localised regions where the microstructure varies rapidly, creating striking patterns in transmitted polarised

* School of Mathematics, University of Bristol, University Walk, Bristol, BS8 1TW, UK

${ }^{\dagger}$ IKERBASQUE, Basque Foundation for Science, Maria Diaz de Haro 3, 48013, Bilbao, Bizkaia, Spain

${ }_{\ddagger}$ BCAM, Basque Center for Applied Mathematics, Mazarredo 14, E48009 Bilbao, Basque Country, Spain

$\S$ "Simion Stoilow" Institute of the Romanian Academy, 21 Calea Grivityei, 010702 Bucharest, Romania 
light. An account of defects is a basic challenge for any theoretical description of nematic liquid crystals $[7,10]$.

In the continuum setting, the material is described by functions taking values in an order-parameter space, which describes the microstructure. The choice of order-parameter space varies between models. In the simplest model, the Oseen-Frank theory, the orderparameter space is the unit sphere $\mathbb{S}^{2}$ with the unit-length vector, $n(x)$, representing the mean local orientation of the molecules at any point $x$. Equilibrium configurations correspond to minimisers of an energy described by a local energy density $\sigma(n, \nabla n)$. Defects correspond to discontinuities in $n$, whose existence may be enforced, for topological reasons, by boundary conditions. Point defects have finite energy in three dimensions, but point defects in two dimensions (or line defects in three dimensions) have infinite energy; this is one of the limitations of the Oseen-Frank theory.

A more refined (and thus more complicated) model is provided by the Landau-de Gennes theory. Here, the order-parameter space is the set of traceless symmetric $3 \times 3$ matrices, referred to in this context as $Q$-tensors, which describe the second moments of the orientational probability distribution for the solute particles (see, eg, [31]). Equilibrium configurations $Q(x)$ are minimisers of an energy described by a local energy density $f(Q, \nabla Q)$. The Oseen-Frank theory emerges in the physically relevant regime where $Q$ has (nearly) everywhere a (nearly) doubly degenerate eigenvalue of fixed magnitude; $Q$ is then (nearly) determined by the orientation of its unique nondegenerate eigenvector, which corresponds to the Oseen-Frank director $n$. Defects correspond to regions where $Q$ is far from having a doubly degenerate eigenvalue. The energy of these regions is finite, and the Landau-de Gennes theory provides a resolution of the discontinuities in the Oseen-Frank model into smooth localised spatial profiles.

The analytic description of defects in the Landau-de Gennes theory is a formidable mathematical challenge. Nevertheless, there has been substantial progress in recent years in analysing the existence, structure, stability and instability of certain defects in three dimensions $[2,3,18,20,26,27,29]$ and two dimensions $[4,6,11,13,14,16,17,21,22]$. These studies have focussed almost exclusively on a simplified version of the Landau-de Gennes theory, in which the elastic (ie, $\nabla Q$-dependent) contribution to the energy density consists solely of the Dirichlet energy - this is the so-called one-constant approximation. It is well known that symmetry considerations allow for additional elastic energy terms.

Here we undertake an exploration of defects in the Landau-de Gennes theory in twodimensional domains when the one-constant restriction is relaxed. Mathematically, this introduces new challenges: the Euler-Lagrange equations become a genuine system of PDEs, and many basic techniques valid for scalar equations fail (though some indeed survive in the two-dimensional setting, see for instance $[4,5,23]$ ). 


\subsection{Isotropy in the Oseen-Frank and Landau-de Gennes theories}

In the absence of a microscopic derivation, the energy in a continuum theory is generally chosen to be i) compatible with underlying symmetries, and ii) as simple as possible while providing a good description of observed phenomena. For nematic liquid crystals, the energy is generally expressed in terms of a local density which, in the absence of external fields, depends only on the order parameter and its first spatial derivatives (and is thereby compatible with translational symmetry in physical space), and contains terms up to quadratic order in the first derivatives. (For problems with free boundary conditions, a surface energy may also be introduced.)

Symmetry under rotations and reflections, which generate the full orthogonal group $O(3)$, imposes additional restrictions on the energy density. The orthogonal group acts on physical space as $x \mapsto P x$, where $P \in O(3)$, and separately on order-parameter space according to the theory. For the Oseen-Frank theory, the $O(3)$-action is given by $n \mapsto P n$, and for the Landau-de-Gennes theory, by $Q \mapsto P Q P^{t}$.

If the energy density is required to be separately invariant under the $O(3)$-actions on physical space and order-parameter space, we obtain its simplest and most restricted form. For the Oseen-Frank theory, this requirement implies that the energy density $\sigma(n, \nabla n)$ is proportional to the Dirichlet energy $n_{i, j} n_{i, j}{ }^{1}$ For the Landau-de Gennes theory, we first decompose the energy density $\psi(Q, \nabla Q)$ into a sum of a bulk term, which depends only on $Q$, and an elastic term, which depends on $Q$ and $\nabla Q$, as follows [1]:

$$
\psi(Q, \nabla Q)=\underbrace{\psi(Q, 0)}_{:=f_{B}(Q) \text { bulk part }}+\underbrace{\psi(Q, \nabla Q)-\psi(Q, 0)}_{:=f_{E}(Q, \nabla Q) \text { elastic part }} .
$$

Invariance under the separate $O(3)$-actions implies that the elastic energy density $f_{E}$ is proportional to the Dirichlet energy $\mathcal{I}_{1}=|\nabla Q|^{2}=Q_{i j, k} Q_{i j, k}$. The bulk energy density is necessarily a function of the spectral invariants $\operatorname{tr}\left(Q^{2}\right)$ and $\operatorname{tr}\left(Q^{3}\right)$ (recall that $\operatorname{tr} Q=0$ ), and is typically taken to be (see, eg, [31])

$$
f_{B}(Q)=-\frac{a^{2}}{2} \operatorname{tr}\left(Q^{2}\right)-\frac{b^{2}}{3} \operatorname{tr}\left(Q^{3}\right)+\frac{c^{2}}{4}\left(\operatorname{tr}\left(Q^{2}\right)\right)^{2} .
$$

Taking the elastic energy to be the Dirichlet energy, in either the Oseen-Frank and Landau-de Gennes theories, leads to considerable simplifications. The second-order differential operator in the Euler-Lagrange equation is just the scalar Laplacian, which does not couple the components of $n$ and $Q$ respectively (coupling occurs only through first-order or zerothorder derivative terms respectively). This enables techniques from scalar PDEs to be carried over to the system of Euler-Lagrange equations.

\footnotetext{
${ }^{1}$ Here and in what follows, we use the summation convention for repeated indices; furthermore we denote $n_{i, j}:=\frac{\partial n_{i}}{\partial x_{j}}, \partial_{k} Q_{i j}=Q_{i j, k}:=\frac{\partial Q_{i j}}{\partial x_{k}}$.
} 
However, there is no physical justification for imposing $O(3)$-invariance separately in physical space and order-parameter space; only joint invariance is required. For the OseenFrank theory, requiring $\sigma(n, \nabla n)$ to be invariant under $n(x) \mapsto P n\left(P^{-1} x\right)$ implies that the energy density is of the following form (see, eg, Section 3.2 of Chapter 3 in [34]) :

$\sigma(n, \nabla n)=K_{1}|\nabla \cdot n|^{2}+K_{2}|n \cdot(\nabla \times n)|^{2}+K_{3}|n \times(\nabla \times n)|^{2}+\left(K_{2}+K_{4}\right)\left[\operatorname{tr}(\nabla n)^{2}-(\nabla \cdot n)^{2}\right]$.

The Oseen-Frank energy (1.2) is found to explain a wide variety of equilibrium phenomena in nematic liquid crystals. (Requiring only $S O(3)$-invariance allows for an additional term linear in $\nabla n$, which can describe cholesteric liquid crystals). The elastic constants $K_{1}, K_{2}, K_{3}$ and $K_{4}$, which are free parameters in the energy density, have been measured in experiments for a variety of materials as well as calculated through numerical simulations.

The term $\operatorname{tr}(\nabla n)^{2}-(\nabla \cdot n)^{2}$ in (1.2), called the saddle-splay term, plays the role of a null Lagrangian; it can be expressed as the pure divergence $\nabla \cdot((n \cdot \nabla) n-(\nabla \cdot n) n)$, whose volume integral depends only on $n$ and its tangential derivatives on the boundary. If the boundary conditions are fixed, the saddle-splay term can be ignored, at least for the purposes of energy minimisation. The Dirichlet energy is recovered by taking $K_{1}=K_{2}=K_{3}$ and $K_{4}=0$. In general, the elastic constants should be chosen so that the energy density is bounded from below. The resulting constraints, first derived by Ericksen[12], are given by

$$
2 K_{1}>K_{2}+K_{4}>0 .
$$

Similarly for the Landau-de Gennes theory, $O(3)$-invariance requires only that the energy density be invariant under $Q(x) \mapsto P Q\left(P^{-1} x\right) P^{t}$. The conditions on the bulk energy remain the same, but the elastic energy $f_{E}(Q, \nabla Q)$ may assume a much more general form. Using methods of representation theory and invariant theory, one can construct the linearly independent, $O(3)$-invariant polynomials quadratic in $\nabla Q$ and of order $m \geq 0$ in $Q$. (If one requires only $S O(3)$-invariance, there are additional invariants linear in $\nabla Q$, which describe cholesteric liquid crystals.) The simplest of the invariants quadratic in $\nabla Q$ are those which are independent of $Q$ (ie, $m=0$ ). There are just three, namely the Dirichlet energy $\mathcal{I}_{1}$ and the following two:

$$
\mathcal{I}_{2}=Q_{i k, j} Q_{i j, k}, \quad \mathcal{I}_{3}=Q_{i j, j} Q_{i k, k} .
$$

The elastic energy assumes the form

$$
f_{E}(\nabla Q)=\frac{L_{1}}{2} Q_{i j, k} Q_{i j, k}+\frac{L_{2}}{2} Q_{i k, j} Q_{i j, k}+\frac{L_{3}}{2} Q_{i j, j} Q_{i k, k}
$$

with elastic constants $L_{1}, L_{2}$ and $L_{3}$ as free parameters.

It is well known that $\mathcal{I}_{2}$ and $\mathcal{I}_{3}$ can be combined to give a null Lagrangian, analogous to the saddle-splay term in the Oseen-Frank energy. As this fact appears to be difficult to find in the literature, we state the result formally here and provide a proof in the Appendix. 
LEMMA 1.1 [Null Lagrangian] Let $\Omega \subset \mathbb{R}^{d}, d=2,3$, be a domain with $C^{1}$ boundary. Let $Q \in H^{1}(\Omega)$ and furthermore, if $d=2$, assume that $\frac{\partial Q}{\partial x_{3}} \equiv 0$. Then:

$$
\int_{\Omega}\left(\mathcal{I}_{2}-\mathcal{I}_{3}\right) d x=\int_{\Omega} Q_{i k, j} Q_{i j, k} d x-\int_{\Omega} Q_{i j, j} Q_{i k, k} d x
$$

depends only on $\left.Q\right|_{\partial \Omega} \in H^{\frac{1}{2}}(\partial \Omega)$.

In general, the elastic constants $L_{1}, L_{2}$ and $L_{3}$ should be chosen so that the energy density is bounded from below. The resulting constraints are obtained, for instance, in [28], using invariant theory arguments. A different approach allows to obtain the same result by standard methods. We have thus the following lemma, whose proof is given in the Appendix.

LEMMA 1.2 [Coercivity conditions] Let $\Omega \subset \mathbb{R}^{d}, d=2,3$. Let $Q \in H^{1}\left(\Omega ; \mathscr{S}_{0}\right)$, and furthermore, if $d=2$, assume $\frac{\partial Q}{\partial x_{3}} \equiv 0$.

1. There exists $\mu_{0}>0$, independent of $Q$, such that for almost all $x \in \Omega$

$$
\frac{L_{1}}{2} Q_{i j, k} Q_{i j, k}+\frac{L_{2}}{2} Q_{i k, j} Q_{i j, k}+\frac{L_{3}}{2} Q_{i j, j} Q_{i k, k} \geq \mu_{0}|\nabla Q|^{2}
$$

if and only if

$$
L_{1}+L_{2}>0,2 L_{1}-L_{2}>0, L_{1}+\frac{L_{2}}{6}+\frac{5}{3} L_{3}>0
$$

2. Let $M:=\frac{L_{1}+L_{2}}{2}$ and $Q_{b} \in H^{\frac{1}{2}}\left(\partial \Omega ; \mathscr{S}_{0}\right)$. Assume that

$$
L_{1}+\frac{4}{3} M>0, \quad L_{1}>0
$$

Then there exists $\mu_{1}>0$ so that for any $Q \in H^{1}\left(\Omega ; \mathscr{S}_{0}\right)$ with $Q=Q_{b}$ we have:

$$
\int_{\Omega} \frac{L_{1}}{2} Q_{i j, k} Q_{i j, k}+M Q_{i j, j} Q_{i k, k} \geq \int_{\Omega} \mu_{1}|\nabla Q|^{2} d x+\mathcal{B}
$$

with $\mathcal{B}$ a constant depending only on $Q_{b}, L_{2}$ and $L_{3}$, but independent of $Q$.

One can connect the $Q$-tensor theory and the Oseen-Frank theory by taking $Q$ to be of the uniaxial form

$$
Q=s_{+}\left(n \otimes n-\frac{1}{3} I\right)
$$

with $n \in S^{2}$ and fixed scalar order parameter $s_{+}$. Some rigorous justifications for this are provided in $[29,32]$ with a physical perspective available in [15]. The constraints are motivated 
by the fact that uniaxial $Q$-tensors with $s_{+}$appropriately chosen are precisely the (degenerate) global minimizers of the bulk potential $f_{B}$ given by (1.1). In a regime where, after suitable nondimensionalization, the elastic constants may be regarded as small, minimizers of the Landau-de Gennes energy approach the uniaxial form (1.9) nearly everywhere.

For uniaxial $Q$-tensors (1.9), the transformation $Q(x) \mapsto P Q\left(P^{-1} x\right) P^{t}$ is equivalent to $n(x) \mapsto P n\left(P^{-1} x\right)$ for $P \in O(3)$. It follows that the Landau-de Gennes elastic energy density $f_{E}$ evaluated on uniaxial $Q$-tensors yields an expression in $n$ and $\nabla n$ of the OseenFrank form (1.2). Straightforward calculations lead to the following identifications of the resulting Oseen-Frank elastic constants in terms of the Landau-de Gennes elastic constants (for simplicity $s_{+}=1$ ):

$$
K_{1}=K_{3}=L_{1}+\frac{1}{2}\left(L_{2}+L_{3}\right), \quad K_{2}=L_{1}, \quad K_{2}+K_{4}=L_{1}+\frac{1}{2} L_{2} .
$$

Thus, for uniaxial $Q$ tensors with fixed scalar order parameter, the Landau-de Gennes elastic energy (1.4) reduces to a restricted form of the Oseen-Frank energy, in which $K_{1}=K_{3}$ (that there must be a restriction is already evident from the fact that the Oseen-Frank elastic energy (1.2) has four elastic constants while the Landau-de Gennes elastic energy (1.4) has only three). The restriction can be overcome by allowing the next-most-simple set of terms in the Landau-de Gennes elastic energy, namely terms quadratic in $\nabla Q$ and linear in $Q$ (ie, $m=1$ above). This introduces additional complications. First, there are six such terms (including an additional null Lagrangian), leading to a surfeit of elastic constants as compared to the Oseen-Frank theory rather than a deficit. Second, the resulting elastic energy density is unbounded below. The latter problem can be addressed by introducing yet more elastic terms, say quadratic in both $Q$ and $\nabla Q$, and/or by modifying the bulk energy (1.1) so as to be infinite outside a compact subset of $Q$-tensors (such modifications are independently motivated by considerations of the microscopic model - see [2], [24] in $d=3$ and [13] in $d=2$ ). In any case, we may conclude that if there are phenomena well described by the Oseen-Frank theory in which the fidelity of the description (qualitative or quantitative) depends on having $K_{1} \neq K_{3}$, then the several-elastic-constant Landau-de Gennes elastic energy (1.4), which is the subject of this paper, may not provide an adequate description.

In the remainder of this paper we will focus on studying the effects induced by the presence of the anisotropic elastic energy terms, $\mathcal{I}_{2}$ and $\mathcal{I}_{3}$, on a basic model problem that is well understood in the case of one elastic constant, namely the two-dimensional k-radially symmetric solutions, studied for instance in $[11,21,22]$.

\subsection{Mathematical formulation of the problem and main results}

As we will be imposing Dirichlet boundary conditions, in view of Lemma 1.2 it suffices to restrict the elastic energy to have just two independent constants, namely $L:=L_{1}$ and 
$M:=\left(L_{2}+L_{3}\right) / 2$. We therefore consider the following form of the Landau-de Gennes energy:

$$
\mathscr{F}[Q ; \Omega]=\int_{\Omega}\left[\frac{L}{2} Q_{i j, k} Q_{i j, k}+M Q_{i j, k} Q_{i k, j}+f_{B}(Q)\right] d x,
$$

where $L, M$ are the independent elastic constants with $L>0, L+\frac{4}{3} M>0$, and $Q \in$ $H^{1}\left(\Omega, \mathscr{S}_{0}\right)$ takes values in the space of $Q$-tensors,

$$
\mathscr{S}_{0} \stackrel{\text { def }}{=}\left\{Q \in \mathbb{R}^{3 \times 3}, Q=Q^{t}, \operatorname{tr}(Q)=0\right\},
$$

The bulk potential $f_{B}(Q)$ is taken to be of the form (1.1) where $a^{2}, b^{2} \geq 0$ and $c^{2}>0$ are material constants. Here and in what follows, summations are taken over $i, j, k=1,2,3$. Since we work in a two-dimensional domain, we assume throughout $Q_{i j, 3} \equiv 0, \forall i, j=1,2,3$. The relationship between the $2 D$ and $3 D$ solutions is discussed in the Appendix, Section 6.3.

We are interested in studying two-dimensional point defects in liquid crystals and their characteristic symmetry features. The simplest and most generic liquid crystal point defects are obtained as critical points of (1.11) in the domain $\Omega=B_{R} \subset \mathbb{R}^{2}$ (the ball of radius $R>0$ centered at the origin) under the following boundary conditions (for more details see $[11]):$

$$
Q(x)=Q_{k}(x) \equiv s_{+}\left(n(x) \otimes n(x)-\frac{1}{3} I\right), x \in \partial B_{R}(0),
$$

where $x=(R \cos \varphi, R \sin \varphi)$,

$$
n(x)=\left(\cos \left(\frac{k}{2} \varphi\right), \sin \left(\frac{k}{2} \varphi\right), 0\right), k \in \mathbb{Z} \backslash\{0\},
$$

and

$$
s_{+}=\frac{b^{2}+\sqrt{b^{4}+24 a^{2} c^{2}}}{4 c^{2}} .
$$

The value of $s_{+}$is chosen so that $Q_{k}$ minimizes $f_{B}(Q)$.

The critical points of the energy (1.11) satisfy the following Euler-Lagrange equations:

$$
\begin{aligned}
L \Delta Q_{i j} & +M\left(\partial_{j} \partial_{k} Q_{i k}+\partial_{i} \partial_{k} Q_{j k}-\frac{2}{3} \partial_{l} \partial_{k} Q_{l k} \delta_{i j}\right) \\
& =-a^{2} Q_{i j}-b^{2}\left(Q_{i k} Q_{k j}-\frac{1}{3}|Q|^{2} \delta_{i j}\right)+c^{2} Q_{i j}|Q|^{2}, i, j=1,2,3 \quad \text { in } B_{R},
\end{aligned}
$$

subject to the boundary conditions (1.13). We note that terms $-\frac{2 M}{3} \partial_{l} \partial_{k} Q_{l k} \delta_{i j}$ and $\frac{b^{2}}{3}|Q|^{2} \delta_{i j}$ account for the constraint $\operatorname{tr}(Q)=0$.

We are interested in studying the critical points compatible with the symmetry of the problem (1.16), (1.13). 
Definition 1.3 For $k \in \mathbb{Z} \backslash\{0\}$, we say that a Lebesgue measurable map $Q: B_{R} \rightarrow \mathscr{S}_{0}$ is generally $k$-radially symmetric if the following condition holds for almost every $x=$ $\left(x_{1}, x_{2}\right) \in B_{R}$ :

$$
Q\left(P_{2}\left(\mathcal{R}_{2}(\psi) \tilde{x}\right)\right)=\mathcal{R}_{k}(\psi) Q(x) \mathcal{R}_{k}^{t}(\psi), \text { for almost every } \psi \in \mathbb{R},
$$

where $\tilde{x}=\left(x_{1}, x_{2}, 0\right), P_{2}: \mathbb{R}^{3} \rightarrow \mathbb{R}^{2}$ is the projection given by $P_{2}\left(x_{1}, x_{2}, x_{3}\right)=\left(x_{1}, x_{2}\right)$, and

$$
\mathcal{R}_{k}(\psi):=\left(\begin{array}{ccc}
\cos \left(\frac{k}{2} \psi\right) & -\sin \left(\frac{k}{2} \psi\right) & 0 \\
\sin \left(\frac{k}{2} \psi\right) & \cos \left(\frac{k}{2} \psi\right) & 0 \\
0 & 0 & 1
\end{array}\right)
$$

represents the rotation about $e_{3}$ by angle $\frac{k}{2} \psi$.

We recall that in [21] we studied a class of critical points of Landau-de Gennes energy with restricted symmetry, namely:

Definition 1.4 For $k \in \mathbb{Z} \backslash\{0\}$, we say that a Lebesgue measurable map $Q: B_{R} \rightarrow \mathscr{S}_{0}$ is $k$-radially symmetric if it is generally $k$-radially symmetric and the following additional condition holds:

(H1) The vector $e_{3}=(0,0,1)$ is an eigenvector of $Q(x)$, for almost all $x \in B_{R}$.

REMARK 1.5 If $k$ is an odd integer, then a generally $k$-radially symmetric $Q \in H^{1}\left(\Omega, \mathscr{S}_{0}\right)$ automatically verifies $(\mathbf{H 1})$, so there is no difference between the two types of symmetries (see Proposition 2.1 in [21]).

In the case $M=0$, all $k$-radially symmetric critical points of Landau-de Gennes energy (1.11) with boundary condition (1.13) have the following form [11, 21, 22]

$$
Y_{k}=u(r) \sqrt{2}\left(n \otimes n-\frac{1}{2} I_{2}\right)+v(r) \sqrt{\frac{3}{2}}\left(e_{3} \otimes e_{3}-\frac{1}{3} I_{3}\right),
$$

where $e_{i}, i=1,2,3$ are the standard cartesian basis vectors, $I_{2}=e_{1} \otimes e_{1}+e_{2} \otimes e_{2}, I_{3}=$ $I_{2}+e_{3} \otimes e_{3}$ and $(u, v)$ satisfies the following system of ODEs:

$$
\begin{aligned}
u^{\prime \prime}+\frac{u^{\prime}}{r}-\frac{k^{2} u}{r^{2}} & =\frac{u}{L}\left[-a^{2}+\sqrt{\frac{2}{3}} b^{2} v+c^{2}\left(u^{2}+v^{2}\right)\right] \\
v^{\prime \prime}+\frac{v^{\prime}}{r} & =\frac{v}{L}\left[-a^{2}-\frac{1}{\sqrt{6}} b^{2} v+c^{2}\left(u^{2}+v^{2}\right)\right]+\frac{1}{\sqrt{6} L} b^{2} u^{2}
\end{aligned}
$$


with boundary conditions

$$
u(0)=0, v^{\prime}(0)=0, u(R)=\frac{1}{\sqrt{2}} s_{+}, v(R)=-\frac{1}{\sqrt{6}} s_{+} .
$$

Moreover, it has been shown that

- in the case $b=0$, the $k$-radially symmetric critical points on $B_{R}(R<\infty)$ are global minimizers of (1.11), (1.13) for any $k \in \mathbb{Z} \backslash\{0\}$ (see [11]);

- in the case $b \neq 0$ all $k$-radially symmetric critical points on $\mathbb{R}^{2}$ are unstable for $k \neq \pm 1$ (see [21]), while for $k= \pm 1$, there exists solutions (1.18) with $u>0$ and $v<0$ on $(0, R)$ with $R \leq \infty$ that are stable.

The main aim of this paper is to study the existence and behaviour of generally k-radially symmetric critical points as well as nonsymmetric local minimisers of the Landau-de Gennes energy (1.11) with boundary conditions (1.13) for general $L$ and $M$ subject to the coercivity conditions (1.7). We also investigate the symmetry-breaking of radial critical points for small nonzero $M$.

We denote, for $\varphi \in[0,2 \pi)$,

$$
n=\left(\cos \left(\frac{k}{2} \varphi\right), \sin \left(\frac{k}{2} \varphi\right), 0\right), m=\left(-\sin \left(\frac{k}{2} \varphi\right), \cos \left(\frac{k}{2} \varphi\right), 0\right) .
$$

We endow the space $\mathscr{S}_{0}$ of $Q$-tensors with the scalar product

$$
Q \cdot \tilde{Q}=\operatorname{tr}(Q \tilde{Q})
$$

and for any $\varphi \in[0,2 \pi)$, we define the following orthonormal basis in $\mathscr{S}_{0}$ :

$$
\begin{gathered}
E_{0}=\sqrt{\frac{3}{2}}\left(e_{3} \otimes e_{3}-\frac{1}{3} I_{3}\right), \\
E_{1}=E_{1}(\varphi)=\sqrt{2}\left(n \otimes n-\frac{1}{2} I_{2}\right), \quad E_{2}=E_{2}(\varphi)=\frac{1}{\sqrt{2}}(n \otimes m+m \otimes n), \\
E_{3}=\frac{1}{\sqrt{2}}\left(n \otimes e_{3}+e_{3} \otimes n\right), \quad E_{4}=\frac{1}{\sqrt{2}}\left(m \otimes e_{3}+e_{3} \otimes m\right) .
\end{gathered}
$$

It is straightforward to check that

$$
\frac{\partial E_{1}}{\partial \varphi}=k E_{2}, \quad \frac{\partial E_{2}}{\partial \varphi}=-k E_{1}, \quad \frac{\partial E_{3}}{\partial \varphi}=\frac{k}{2} E_{4}, \quad \frac{\partial E_{4}}{\partial \varphi}=-\frac{k}{2} E_{3} .
$$

REMARK 1.6 The above basis is relevant only for the case when $k$ is even. For $k$ odd, $E_{3}$ and $E_{4}$ are not $2 \pi$-periodic in $\varphi$. In this case, a good basis can be obtained as in [21] by replacing $E_{3}$ with $\tilde{E}_{3}=\frac{1}{\sqrt{2}}\left(e_{1} \otimes e_{3}+e_{3} \otimes e_{1}\right)$ and $E_{4}$ with $\tilde{E}_{4}=\frac{1}{\sqrt{2}}\left(e_{2} \otimes e_{3}+e_{3} \otimes e_{2}\right)$. 
Our main analytical result provides conditions for the existence of generally $k$-radially symmetric critical points of the Landau-de Gennes energy.

Theorem 1.7 Assume that $k \in \mathbb{Z} \backslash\{0\}, L>0, M \neq 0$, and $L+\frac{4}{3} M>0$.

1. If $k \neq 2$ then there exist no generally $k$-radially symmetric critical points of the Landaude Gennes energy (1.11) subject to boundary conditions (1.13).

2. If $k=2$ then $Q \in H^{1}\left(B_{R}, \mathscr{S}_{0}\right)$ is a generally $k$-radially symmetric critical point of the Landau-de Gennes energy (1.11) satisfying the boundary conditions (1.13) if and only if

$$
Q(x)=\sum_{i=0}^{4} w_{i}(r) E_{i},
$$

where $w_{i} \in C^{\infty}(0, R)$ satisfy the following system of $O D E$ 's:

$$
\begin{aligned}
& (L+M / 3)\left(w_{0}^{\prime \prime}+\frac{w_{0}^{\prime}}{r}\right)-\frac{M}{\sqrt{3}}\left(w_{1}^{\prime \prime}+\frac{3 w_{1}^{\prime}}{r}\right) \\
& =w_{0}\left(-a^{2}-\frac{b^{2}}{\sqrt{6}} w_{0}+c^{2}\left(\sum_{i=0} w_{i}^{2}\right)\right)+\frac{b^{2}}{\sqrt{6}}\left(w_{1}^{2}+w_{2}^{2}\right)-\frac{b^{2}}{2 \sqrt{6}}\left(w_{3}^{2}+w_{4}^{2}\right), \\
& (L+M)\left(w_{1}^{\prime \prime}+\frac{w_{1}^{\prime}}{r}-\frac{4 w_{1}}{r^{2}}\right)-\frac{M}{\sqrt{3}}\left(w_{0}^{\prime \prime}-\frac{w_{0}^{\prime}}{r}\right) \\
& \quad=w_{1}\left(-a^{2}+\frac{2 b^{2}}{\sqrt{6}} w_{0}+c^{2}\left(\sum_{i=0}^{4} w_{i}^{2}\right)\right)-\frac{b^{2}}{2 \sqrt{2}}\left(w_{3}^{2}-w_{4}^{2}\right), \\
& (L+M)\left(w_{2}^{\prime \prime}+\frac{w_{2}^{\prime}}{r}-\frac{4 w_{2}}{r^{2}}\right)=w_{2}\left(-a^{2}+\frac{2 b^{2}}{\sqrt{6}} w_{0}+c^{2}\left(\sum_{i=0}^{4} w_{i}^{2}\right)\right)-\frac{b^{2}}{\sqrt{2}} w_{3} w_{4}, \\
& (L+M)\left(w_{3}^{\prime \prime}+\frac{w_{3}^{\prime}}{r}-\frac{w_{3}}{r^{2}}\right)=w_{3}\left(-a^{2}-\frac{b^{2}}{\sqrt{6}} w_{0}-\frac{b^{2}}{\sqrt{2}} w_{1}+c^{2}\left(\sum_{i=0}^{4} w_{i}^{2}\right)\right)-\frac{b^{2}}{\sqrt{2}} w_{2} w_{4}, \\
& L\left(w_{4}^{\prime \prime}+\frac{w_{4}^{\prime}}{r}-\frac{w_{4}}{r^{2}}\right)=w_{4}\left(-a^{2}-\frac{b^{2}}{\sqrt{6}} w_{0}+\frac{b^{2}}{\sqrt{2}} w_{1}+c^{2}\left(\sum_{i=0}^{4} w_{i}^{2}\right)\right)-\frac{b^{2}}{\sqrt{2}} w_{2} w_{3},
\end{aligned}
$$

subject to the boundary conditions

$$
\begin{aligned}
& w_{0}^{\prime}(0)=0, w_{1}(0)=0, w_{2}(0)=0, w_{3}(0)=0, w_{4}(0)=0 \\
& w_{0}(R)=-\frac{s_{+}}{\sqrt{6}}, w_{1}(R)=\frac{s_{+}}{\sqrt{2}}, w_{2}(R)=0, w_{3}(R)=0, w_{4}(R)=0 .
\end{aligned}
$$


Using this result, in Section 3 we carry out a numerical study of the generally $k$-radially symmetric solutions for $k=2$. Depending on the elastic constant $M$, the material parameter $b$ and the radius $R$, we observe three types of solutions, which we classify in terms of the number of non-vanishing coefficients $w_{i}$, as follows:

- two-component solutions, where $Q=w_{0}(r) E_{0}+w_{1}(r) E_{1}$;

- three-component solutions, where $Q=w_{0}(r) E_{0}+w_{1}(r) E_{1}+w_{3}(r) E_{3}$;

- five-component solutions where $Q=\sum_{i=0}^{4} w_{i}(r) E_{i}$.

These solutions appear as minimizers (local or global) of the full Landau-de Gennes energy in certain parameter regimes. We determine the profiles and stability properties of these solutions, and discuss a number of their characteristic features, including symmetries, the monotonicity and signs of the radial components. We also find non-radially-symmetric solutions with $\mathbb{Z}_{2}$ symmetry, qualitatively similar to previously reported solutions [4, 17], exhibiting two separated index-one-half defects. By comparing the (numerically determined) energies of all these solutions, we obtain the global phase diagram.

In the Section 4 we investigate how general $k$-radial symmetry is broken for small $M \neq 0$ in the physically interesting cases $k= \pm 1$. Using formal asymptotics, we characterise the leading-order symmetry-breaking terms as solutions of a system of linear inhomogeneous ODE's generated by the second variation of the Landau-de Gennes energy. These asymptotic results are supported by numerical calculations.

In the last section of the paper we present a number of open problems (both analytical and numerical) motivated by our numerical investigations. It is hoped that these will stimulate the development of new techniques for addressing the physically relevant but mathematically challenging Landau-de Gennes model with several elastic constants. Finally, in the Appendix we cover a number of technical questions necessary for the paper.

\section{Radially symmetric critical points}

This section is devoted to an analytical study of generally $k$-radially symmetric critical points of the Landau-de Gennes energy (1.11) subject to the boundary conditions (1.13). In particular, we show that if $M \neq 0$, then generally $k$-radially symmetric critical points do not exist if $k \neq 2$. In the case $k=2$ we derive the system of ODEs satisfied by generally $k$-radially symmetric critical points and using variational methods prove the existence of a solution for this system. Finally, we study the interesting limit $M \rightarrow \infty$.

We begin with the proof of Theorem 1.7 about existence of generally $k$-radially symmetric critical points. 
Proof of Theorem 1.7. We know that any generally $k$-radially symmetric $Q$-tensor $Q \in$ $H^{1}\left(B_{R} ; \mathscr{S}_{0}\right)$ can be represented in the form

$$
Q=\sum_{i=0}^{4} w_{i}(r) E_{i}
$$

where $w_{0} \in H^{1}((0, R) ; r d r)$ and $w_{i} \in H^{1}((0, R) ; r d r) \cap L^{2}\left((0, R) ; \frac{1}{r} d r\right)$ for $1 \leq i \leq 4$. Moreover, for $k$ odd, the components $w_{3}, w_{4}$ vanish, see Proposition 2.1 in [21].

Similarly as in [21] we prove now that $w_{i}(0)=0$ for $1 \leq i \leq 4$. Since $w_{i} \in H^{1}((0, R) ; r d r) \cap$ $L^{2}\left((0, R) ; \frac{1}{r} d r\right)$ we have that that $w_{i}$ is continuous on $(0, R)$ and for $r_{1}, r_{2} \in(0, R)$ :

$$
\left|w_{i}^{2}\left(r_{2}\right)-w_{1}^{2}\left(r_{1}\right)\right|=2\left|\int_{r_{1}}^{r_{2}} w_{i} w_{i}^{\prime} d r\right| \leq 2\left(\int_{r_{1}}^{r_{2}} w_{i}^{2} \frac{d r}{r}\right)^{1 / 2}\left(\int_{r_{1}}^{r_{2}}\left(w_{i}^{\prime}\right)^{2} r d r\right)^{1 / 2} .
$$

Since the right hand side converges to zero as $\left|r_{2}-r_{1}\right| \rightarrow 0$, it follows that $w_{i}$ is continuous up to $r=0$. Combined again with $w_{i} \in L^{2}\left((0, R) ; \frac{1}{r} d r\right)$, we conclude that $w_{i}(0)=0$.

We would like to understand under what conditions the generally $k$-radially symmetric $Q$-tensor satisfies the full Euler-Lagrange equations (1.16) with boundary conditions (1.13). The nonlinear part, contained in the right-hand side of the Euler-Lagrange equations (1.16), becomes

$$
\begin{aligned}
-a^{2} Q-b^{2} & \left(Q^{2}-\frac{1}{3}|Q|^{2} I\right)+c^{2} Q|Q|^{2}= \\
& {\left[w_{0}\left(-a^{2}-\frac{2 b^{2}}{\sqrt{6}} w_{0}+c^{2}\left(\sum_{i=0}^{4} w_{i}^{2}\right)\right)+\frac{b^{2}}{\sqrt{6}}\left(\sum_{i=0}^{4} w_{i}^{2}\right)-\frac{3 b^{2}}{2 \sqrt{6}}\left(w_{3}^{2}+w_{4}^{2}\right)\right] E_{0} } \\
& +\left[w_{1}\left(-a^{2}+\frac{2 b^{2}}{\sqrt{6}} w_{0}+c^{2}\left(\sum_{i=0}^{4} w_{i}^{2}\right)\right)-\frac{b^{2}}{2 \sqrt{2}}\left(w_{3}^{2}-w_{4}^{2}\right)\right] E_{1} \\
+ & {\left[w_{2}\left(-a^{2}+\frac{2 b^{2}}{\sqrt{6}} w_{0}+c^{2}\left(\sum_{i=0}^{4} w_{i}^{2}\right)\right)-\frac{b^{2}}{\sqrt{2}} w_{3} w_{4}\right] E_{2} } \\
+ & {\left[w_{3}\left(-a^{2}-\frac{b^{2}}{\sqrt{6}} w_{0}-\frac{b^{2}}{\sqrt{2}} w_{1}+c^{2}\left(\sum_{i=0}^{4} w_{i}^{2}\right)\right)-\frac{b^{2}}{\sqrt{2}} w_{2} w_{4}\right] E_{3} } \\
+ & {\left[w_{4}\left(-a^{2}-\frac{b^{2}}{\sqrt{6}} w_{0}+\frac{b^{2}}{\sqrt{2}} w_{1}+c^{2}\left(\sum_{i=0}^{4} w_{i}^{2}\right)\right)-\frac{b^{2}}{\sqrt{2}} w_{2} w_{3}\right] E_{4} }
\end{aligned}
$$

with $w_{3}=w_{4}=0$ in the case of odd $k$.

The elastic part, contained in the left-hand side of the Euler-Lagrange equations (1.16), provides an elliptic operator under the assumed conditions on $L$ and $M$. Standard arguments 
(see for instance [9]) show that the solutions are smooth. Then one can easily check that

$$
w_{i} \in C^{\infty}(0, R)
$$

for $i=0, \ldots 4$ as claimed.

Furthermore, since $Q$ is smooth in $B_{R}$, we obtain that $\operatorname{tr}\left(Q E_{0}\right)$ is smooth in $B_{R}$ and in particular along the line $\{(x, 0) ;-R \leq x \leq R\}$. Noting that $w_{0}(r)=\operatorname{tr}\left(Q(r, 0) E_{0}\right)$ for $r \geq 0$ and taking into account that $Q$ is radially symmetric, we can trivially extend $w_{0}$ to $(-R, R)$ in an even and smooth way. Therefore $w_{0} \in C^{\infty}([0, R))$ and $w_{0}^{\prime}(0)=0$.

Furthermore the elastic part generates a linear operator. Therefore, we can study the action of the elastic part on the components $w_{i}(r) E_{i}$ separately. For convenience, we define the operator

$$
\mathcal{L} Q_{i j}=\partial_{j} \partial_{k} Q_{i k}+\partial_{i} \partial_{k} Q_{j k}-\frac{2}{3} \partial_{l} \partial_{k} Q_{l k} \delta_{i j}
$$

We notice that

$$
(L \Delta+M \mathcal{L})\left(\sum_{i=0}^{2} w_{i}(r) E_{i}\right) \in \operatorname{span}\left\{E_{0}, E_{1}, E_{2}\right\}
$$

and

$$
(L \Delta+M \mathcal{L})\left(\sum_{i=3}^{4} w_{i}(r) E_{i}\right) \in \operatorname{span}\left\{E_{3}, E_{4}\right\}
$$

with the convention that $w_{3}=w_{4}=0$ when $k$ is odd.

Let us first compute the elastic part for (2.3). We note that for any $k \in \mathbb{Z} \backslash\{0\}$ (see [11])

$$
\Delta\left(\sum_{i=0}^{2} w_{i}(r) E_{i}\right)=\left(w_{0}^{\prime \prime}+\frac{w_{0}^{\prime}}{r}\right) E_{0}+\left(w_{1}^{\prime \prime}+\frac{w_{1}^{\prime}}{r}-\frac{k^{2} w_{1}}{r^{2}}\right) E_{1}+\left(w_{2}^{\prime \prime}+\frac{w_{2}^{\prime}}{r}-\frac{k^{2} w_{2}}{r^{2}}\right) E_{2} .
$$

After a lengthy but straightforward calculation (see Appendix, Section 6.4) we also obtain

$$
\begin{aligned}
& \mathcal{L}\left(w_{0}(r) E_{0}\right)=\frac{1}{3}\left(w_{0}^{\prime \prime}+\frac{w_{0}^{\prime}}{r}\right) E_{0}-\frac{1}{\sqrt{3}}\left(w_{0}^{\prime \prime}-\frac{w_{0}^{\prime}}{r}\right)\left(E_{1} \cos ((k-2) \varphi)-E_{2} \sin ((k-2) \varphi)\right), \\
& \mathcal{L}\left(w_{1}(r) E_{1}\right)=\left(w_{1}^{\prime \prime}+\frac{w_{1}^{\prime}}{r}-\frac{k^{2} w_{1}}{r^{2}}\right) E_{1}-\frac{E_{0}}{\sqrt{3}}\left(w_{1}^{\prime \prime}+\frac{(2 k-1) w_{1}^{\prime}}{r}+\frac{k(k-2) w_{1}}{r^{2}}\right) \cos ((k-2) \varphi), \\
& \mathcal{L}\left(w_{2}(r) E_{2}\right)=\left(w_{2}^{\prime \prime}+\frac{w_{2}^{\prime}}{r}-\frac{k^{2} w_{2}}{r^{2}}\right) E_{2}+\frac{E_{0}}{\sqrt{3}}\left(w_{2}^{\prime \prime}+\frac{(2 k-1) w_{2}^{\prime}}{r}+\frac{k(k-2) w_{2}}{r^{2}}\right) \sin ((k-2) \varphi) .
\end{aligned}
$$

Since $E_{0}, E_{1}$ and $E_{2}$ are linearly independent, we see that the only possibility to obtain a closed system of ordinary differential equations for $w_{0}, w_{1}$ and $w_{2}$ is to take $k=2$. Therefore, 
even without computing the terms in (2.4), we may conclude that for any $k \neq 2, M \neq 0$ there is no generally $k$-radially symmetric solution of (1.16).

Now we want to investigate the case $k=2$. We already computed the term $(L \Delta+$ $M \mathcal{L})\left(\sum_{i=0}^{2} w_{i}(r) E_{i}\right)$ and we are left with finding $(L \Delta+M \mathcal{L})\left(\sum_{i=3}^{4} w_{i}(r) E_{i}\right)$. After a straightforward calculation we obtain

$$
(L \Delta+M \mathcal{L})\left(\sum_{i=3}^{4} w_{i}(r) E_{i}\right)=(L+M) E_{3}\left(w_{3}^{\prime \prime}+\frac{w_{3}^{\prime}}{r}-\frac{w_{3}}{r^{2}}\right)+L E_{4}\left(w_{4}^{\prime \prime}+\frac{w_{4}^{\prime}}{r}-\frac{w_{4}}{r^{2}}\right) .
$$

Combining (2.1), (2.5), (2.6), (2.7) we observe that the Euler-Lagrange equations become (1.25). Using boundary conditions (1.13) for the PDE system we obtain the boundary conditions for the ODE at $R$.

In order to prove existence of the solution for the problem (1.25), (1.26) we minimize the corresponding energy functional

$$
\begin{aligned}
\mathscr{E}\left(\left\{w_{i}\right\}\right) & =\int_{0}^{R}\left\{\frac{L}{2}\left(\left|w_{1}^{\prime}\right|^{2}+\left|w_{0}^{\prime}\right|^{2}+\frac{4}{r^{2}} w_{1}^{2}\right)+\frac{M}{6}\left|\sqrt{3} w_{1}^{\prime}-w_{0}^{\prime}+\frac{2 \sqrt{3}}{r} w_{1}\right|^{2}\right\} r d r \\
& +\int_{0}^{R}\left\{\frac{L+M}{2}\left(\left|w_{2}^{\prime}\right|^{2}+\frac{4}{r^{2}} w_{2}^{2}+\left|w_{3}^{\prime}\right|^{2}+\frac{1}{r^{2}} w_{3}^{2}\right)+\frac{L}{2}\left(\left|w_{4}^{\prime}\right|^{2}+\frac{1}{r^{2}} w_{4}^{2}\right)\right\} r d r \\
& +\int_{0}^{R}\left\{\left(-\frac{a^{2}}{2}+\frac{c^{2}}{4}\left(\sum_{i=0}^{4} w_{i}^{2}\right)\right)\left(\sum_{i=0}^{4} w_{i}^{2}\right)\right. \\
& \left.-\frac{b^{2} \sqrt{6}}{36}\left(2 w_{0}^{3}-6 w_{0}\left(w_{1}^{2}+w_{2}^{2}\right)+3 w_{0}\left(w_{3}^{2}+w_{4}^{2}\right)+3 \sqrt{3} w_{1}\left(w_{3}^{2}-w_{4}^{2}\right)+6 \sqrt{3} w_{2} w_{3} w_{4}\right)\right\} r d r
\end{aligned}
$$

defined on the admissible set $S \times\left(S_{w}\right)^{3}$ where $S$ and $S_{w}$ are defined as

$S=\left\{\left(w_{0}, w_{1}\right):[0, R] \rightarrow \mathbb{R}^{2} \mid \sqrt{r} w_{0}^{\prime}, \sqrt{r} w_{1}^{\prime}, \sqrt{r} w_{0}, \frac{w_{1}}{\sqrt{r}} \in L^{2}(0, R), w_{0}(R)=-\frac{s_{+}}{\sqrt{6}}, w_{1}(R)=\frac{s_{+}}{\sqrt{2}}\right\}$

and

$$
S_{w}=\left\{w:[0, R] \rightarrow \mathbb{R} \mid \sqrt{r} w^{\prime}, \frac{w}{\sqrt{r}} \in L^{2}(0, R), w(R)=0\right\} .
$$

It is clear that for $M \geq 0$ the above energy is coercive and therefore existence of a minimizer follows by standard arguments. In the case $M<0$ we can rewrite the energy as

$$
\mathscr{E}\left(\left\{w_{i}\right\}\right)=\frac{2 M s_{+}^{2}}{3}+\int_{0}^{R}\left\{\left(\frac{L}{2}+\frac{4 M}{6}\right)\left(\left|w_{1}^{\prime}\right|^{2}+\left|w_{0}^{\prime}\right|^{2}+\frac{4}{r^{2}} w_{1}^{2}\right)-\frac{M}{6}\left|\sqrt{3} w_{0}^{\prime}+w_{1}^{\prime}+\frac{2}{r} w_{1}\right|^{2}\right\} r d r
$$




$$
\begin{aligned}
& +\int_{0}^{R}\left\{\frac{L+M}{2}\left(\left|w_{2}^{\prime}\right|^{2}+\frac{4}{r^{2}} w_{2}^{2}+\left|w_{3}^{\prime}\right|^{2}+\frac{1}{r^{2}} w_{3}^{2}\right)+\frac{L}{2}\left(\left|w_{4}^{\prime}\right|^{2}+\frac{1}{r^{2}} w_{4}^{2}\right)\right\} r d r \\
& +\int_{0}^{R}\left\{\left(-\frac{a^{2}}{2}+\frac{c^{2}}{4}\left(\sum_{i=0}^{4} w_{i}^{2}\right)\right)\left(\sum_{i=0}^{4} w_{i}^{2}\right)\right. \\
& \left.-\frac{b^{2} \sqrt{6}}{36}\left(2 w_{0}^{3}-6 w_{0}\left(w_{1}^{2}+w_{2}^{2}\right)+3 w_{0}\left(w_{3}^{2}+w_{4}^{2}\right)+3 \sqrt{3} w_{1}\left(w_{3}^{2}-w_{4}^{2}\right)+6 \sqrt{3} w_{2} w_{3} w_{4}\right)\right\} r d r
\end{aligned}
$$

and using the coercivity conditions $L>0, L+\frac{4}{3} M>0$, we can obtain the existence of a minimizer by standard methods.

REMARK 2.1 It is clear from the system (1.25) that one can take $w_{2}=w_{3}=w_{4}=0$ and obtain a special $k$-radially symmetric solution of the full Euler-Lagrange equation (1.16) of the form

$$
Q=w_{0}(r) E_{0}+w_{1}(r) E_{1}
$$

where $\left(w_{0}, w_{1}\right) \in S$ minimizes the following energy (see also the corresponding representation (2.11))

$$
\begin{aligned}
\mathscr{E}\left(w_{0}, w_{1}\right) & =\int_{0}^{R}\left\{\frac{L}{2}\left(\left|w_{1}^{\prime}\right|^{2}+\left|w_{0}^{\prime}\right|^{2}+\frac{4}{r^{2}} w_{1}^{2}\right)+\frac{M}{6}\left|\sqrt{3} w_{1}^{\prime}-w_{0}^{\prime}+\frac{2 \sqrt{3}}{r} w_{1}\right|^{2}\right\} r d r \\
& +\int_{0}^{R}\left\{\left(-\frac{a^{2}}{2}+\frac{c^{2}}{4}\left(w_{0}^{2}+w_{1}^{2}\right)\right)\left(w_{0}^{2}+w_{1}^{2}\right)-\frac{b^{2} \sqrt{6}}{18}\left(w_{0}^{3}-3 w_{0} w_{1}^{2}\right)\right\} r d r
\end{aligned}
$$

and satisfies the following system of ODEs

$$
\begin{aligned}
& \left(L+\frac{M}{3}\right)\left(w_{0}^{\prime \prime}+\frac{w_{0}^{\prime}}{r}\right)-\frac{M}{\sqrt{3}}\left(w_{1}^{\prime \prime}+\frac{3 w_{1}^{\prime}}{r}\right)=w_{0}\left(-a^{2}-\frac{b^{2}}{\sqrt{6}} w_{0}+c^{2}\left(w_{0}^{2}+w_{1}^{2}\right)\right)+\frac{b^{2}}{\sqrt{6}} w_{1}^{2}, \\
& (L+M)\left(w_{1}^{\prime \prime}+\frac{w_{1}^{\prime}}{r}-\frac{4 w_{1}}{r^{2}}\right)-\frac{M}{\sqrt{3}}\left(w_{0}^{\prime \prime}-\frac{w_{0}^{\prime}}{r}\right)=w_{1}\left(-a^{2}+\frac{2 b^{2}}{\sqrt{6}} w_{0}+c^{2}\left(w_{0}^{2}+w_{1}^{2}\right)\right)
\end{aligned}
$$

with boundary conditions

$$
w_{0}^{\prime}(0)=0, w_{1}(0)=0, w_{0}(R)=-\frac{s_{+}}{\sqrt{6}}, w_{1}(R)=\frac{s_{+}}{\sqrt{2}} .
$$

The solution $Q$ will always have $e_{3}$ as an eigenvector, and therefore is 2-radially symmetric according to Definition 1.4 (see also $[11,21]$ ). As shown in Section 3, numerical calculations indicate that generally 2-radially symmetric solutions are not always of the restricted form $(2.12)$. 
We have shown the existence of generally 2-radially symmetric solutions $Q=\sum_{i=0}^{4} w_{i}(r) E_{i}$ for any $L>0$ and $M>-\frac{3}{4} L$. In general, there is no obvious reason for these solutions not to have all five components present. However, when $M$ is large enough, we can show that generally 2-radially symmetric solutions are of a restricted form.

Lemma 2.2 Assume $L>0$. For any fixed $M$ denote by $\mathscr{E}_{M}\left(\left\{w_{i}\right\}\right)$ the energy in $(2.11)$. For $M \rightarrow+\infty$, the following statements hold:

1. If $\mathscr{E}_{M}\left(\left\{w_{i}^{M}\right\}\right) \leq C$ with $C$ independent of $M$ then $w_{0}^{M} \rightarrow w_{0}$ weakly in $H^{1}((0, R) ; r d r)$, $w_{i}^{M} \rightarrow w_{i}$ weakly in $H^{1}((0, R) ; r d r) \cap L^{2}\left((0, R) ; \frac{1}{r} d r\right)$ for $i=0, \ldots, 4$ (maybe for $a$ subsequence) and

$$
w_{2}=w_{3}=0, \sqrt{3}\left(w_{1} r^{2}\right)^{\prime}=r^{2} w_{0}^{\prime} \text { a.e. } r \in(0, R) .
$$

2. The energy $\mathscr{E}_{M} \Gamma$-converges in the weak topology of $H^{1}((0, R) ; r d r) \times\left[H^{1}((0, R) ; r d r) \cap\right.$ $\left.L^{2}\left((0, R) ; \frac{1}{r} d r\right)\right]^{4}$ to:

$$
\mathscr{E}_{\infty}:= \begin{cases}\int_{0}^{R}\left\{\frac{L}{2}\left(\left|w_{1}^{\prime}\right|^{2}+\left|w_{0}^{\prime}\right|^{2}+\left|w_{4}^{\prime}\right|^{2}+\frac{1}{r^{2}}\left(4 w_{1}^{2}+w_{4}^{2}\right)\right)+h\left(w_{0}, w_{1}, w_{4}\right)\right\} r d r & \text { if }(2.16) \text { holds } \\ +\infty & \text { otherwise }\end{cases}
$$

where

$$
\begin{aligned}
h\left(w_{0}, w_{1}, w_{4}\right) & =\left(-\frac{a^{2}}{2}+\frac{c^{2}}{4}\left(w_{0}^{2}+w_{1}^{2}+w_{4}^{2}\right)\right)\left(w_{0}^{2}+w_{1}^{2}+w_{4}^{2}\right) \\
& -\frac{b^{2} \sqrt{6}}{36}\left(2 w_{0}^{3}-6 w_{0} w_{1}^{2}+3 w_{0} w_{4}^{2}-3 \sqrt{3} w_{1} w_{4}^{2}\right) .
\end{aligned}
$$

Proof. We notice that the first statement trivially follows from the uniform energy bounds. In order to show $\Gamma$-convergence we need two statements:

1. If $w_{0}^{M} \rightarrow w_{0}$ in $H^{1}((0, R) ; r d r)$ and $w_{i}^{M} \rightarrow w_{i}$ in $H^{1}((0, R) ; r d r) \cap L^{2}\left((0, R) ; \frac{1}{r} d r\right)$ for $i=1, \ldots, 4$ then

$$
\liminf _{M \rightarrow \infty} \mathscr{E}_{M}\left(\left\{w_{i}^{M}\right\}\right) \geq \mathscr{E}_{\infty}\left(\left\{w_{i}\right\}\right)
$$

2. For any $w_{0} \in H^{1}((0, R) ; r d r)$ and $w_{i} \in H^{1}((0, R) ; r d r) \cap L^{2}\left((0, R) ; \frac{1}{r} d r\right)$ for $i=$ $1, \ldots, 4$ there exists a sequence $w_{0}^{M} \in H^{1}((0, R) ; r d r)$ and $w_{i}^{M} \in H^{1}((0, R) ; r d r) \cap$ $L^{2}\left((0, R) ; \frac{1}{r} d r\right)$ for $i=1, \ldots, 4$ such that

$$
\limsup _{M \rightarrow \infty} \tilde{\mathscr{E}}_{M}\left(w_{i}^{M}\right)=\mathscr{E}_{\infty}\left(w_{i}\right) .
$$

The first statement is just lower semicontinuity of the energy $\mathscr{E}_{M}$ and for the second we take $w_{i}^{M}=w_{i}, i=0, \ldots, 4$. The existence of minimizers for the limiting energy $\mathscr{E}_{\infty}$ follows by standard arguments. 


\section{$3 \quad k=2$ defects}

In this section, we carry out a numerical study of two-dimensional defects for the two-elasticconstant Landau-de Gennes energy (1.11) in the case $k=2$. From Theorem 1.7, it is only for $k=2$ that generally $k$-radially symmetric solutions of the Euler-Lagrange equations exist for general nonzero $M$. We consider the cases $b=0$ and $b=1$ separately in Sections 3.1 and 3.2 respectively. The two cases are distinguished by the set of minimisers of the bulk potential $f_{b}(Q)$, which for $b=0$ is the four-sphere $|Q|^{2}=\frac{a^{2}}{c^{2}}$, while for $b>0$ is the two-dimensional space of uniaxial $Q$-tensors with scalar order parameter $s_{+}$given by (1.15). The numerical results build upon previous analytic results for the case $M=0$ presented in [11] for $b=0$ and $[21,22]$ for $b>0$. Throughout, we fix $L=1$ and $a=c=1$. As explained below, we find global and local minimisers of the Landau-de Gennes energy as well as some, but not necessarily all, of the generally 2-radially symmetric saddle points. We find bifurcations in these solutions as functions of $M$ and $R$, where $R$ is the radius of the disk, and study their characteristics in different parameter regimes.

The numerical procedure consists of finding local minimisers of i) the full energy (1.11), ii) the reduced energy (2.8), which is defined on generally 2-radially symmetric profiles characterised by radial functions $w_{0}(r), \ldots, w_{4}(r)$, and iii) the restriction of the reduced energy obtained by taking only $w_{0}$ and $w_{1}$ to be nonzero, given by (2.13). From Theorem 1.7, it follows that local minimisers found in ii) and iii) are necessarily critical points of the full energy (1.11). By considering the second variation of the full energy, we can determine whether these critical points are local minimisers or saddle points. We also find non-radially-symmetric local minimisers of the full energy. Comparing the energies of all the local minimisers obtained in this way, we identify the global minimiser as the one with the lowest energy. As we are not solving the Euler-Lagrange equation directly, we are not able to find saddle points of the full energy that are not radially symmetric, nor are we able to find radially symmetric critical points that are not minimisers of either the reduced energy (2.8) or its two-component restriction (2.13). We employ a numerical technique of continuation in parameter $M$ similar to one used in [30], where minimisers of the energy for the previous value of $M$ are used as initial trials for the perturbed value of $M$. At each energy minimisation step for (1.11) after discretising $Q$-tensors satisfying boundary condition (1.13) with linear finite elements the resulting discrete energy is minimised using a trust-region method of nonlinear unconstrained optimisation. We restrict our numerical analysis to moderate values of the radius, $5 \leq R \leq 50$ and the elastic constant, $0.75<M \leq 100$.

Following this procedure, we find the following types of radially-symmetric and nonradially-symmetric critical points.

Radially-symmetric:

- Two-component solutions $Q_{2}$ with only $w_{0}$ and $w_{1}$ nonzero. These are the simplest radial solutions, for which $n, m$ and $e_{3}$ are everywhere eigenvectors of the $Q$-tensors. 
We identify two sub-types, namely

$-Q_{2}^{-}$, with $w_{0}$ and $w_{1}$ monotonic, $w_{0}<0$ and $w_{1}>0$. Solutions of this type were studied in detail in [11] for $M=b=0$ and in [21, 22] for $M=0, b \neq 0$.

- $Q_{2}^{ \pm}$, with $w_{0}$ changing sign. In [11], it was shown that for $M=b=0$, a global minimizer of the radial energy cannot be of this type.

- Three-component solution $Q_{3}$, with $w_{0}, w_{1}$ and $w_{3}$ nonzero. Neither $n$ nor $e_{3}$ are everywhere eigenvectors of $Q_{3}$, although $m$ is.

- Five-component solution $Q_{5}$, with no vanishing components. The eigenvectors of $Q_{5}$ do not retain any of the characteristics of the boundary data; neither $n, m$ nor $e_{3}$ are eigenvectors of $Q_{5}$ except at special points (e.g., the origin).

\section{Non-radially-symmetric:}

A non-radially-symmetric solution belongs to a one-parameter family of solutions generated by rotations about $e_{3}$. They are found to have two isolated points where the $Q$-tensor is uniaxial, and about which the $Q$-tensor has the structure of a defect with $k=1$. The defects are collinear with and equidistant from the origin, and for definiteness may be taken to lie along the $e_{1}$-axis. We find two sub-types, namely

- Vertical non-radial solution $Q_{N R}^{v}$, for which $e_{3}$ is everywhere an eigenvector. The solution inherits four discrete symmetries from the boundary data, namely reflection invariance through the $\left(e_{1}, e_{3}\right)$ - and $\left(e_{2}, e_{3}\right)$-planes, and $\pi$-rotation invariance about the $e_{1}$ - and $e_{2}$-axes.

- Tilted non-radial solution $Q_{N R}^{t}$, for which $e_{3}$ is not an eigenvector (except at special points). The solution inherits only two discrete symmetries from the boundary data, namely reflection invariance through the $\left(e_{2}, e_{3}\right)$-plane and $\pi$-rotation invariance about the $e_{1}$-axis.

\subsection{Case $b=0$}

Figure 1 displays a partial phase diagram for the Landau-de Gennes energy as a function of the elastic constant $M$ and radius $R$, following the numerical procedure described above. Global minimisers, local minimisers and saddle points are as indicated. For $M$ sufficiently large (to the right of the blue curve in Figure 1), the global minimiser is radially symmetric of type $Q_{2}^{-}$, in keeping with analytic results for $M=0$ [11]. The radially symmetric solution of type $Q_{2}^{ \pm}$, which for $M=0$ was shown to be saddle point for small $L$ [11], actually becomes the global minimiser for $M$ sufficiently negative. For intermediate values of $M$ (between the magenta, black and green curves), the global minimiser is found to be non-radially symmetric of type $Q_{N R}^{t}$ or $Q_{N R}^{v}$. 


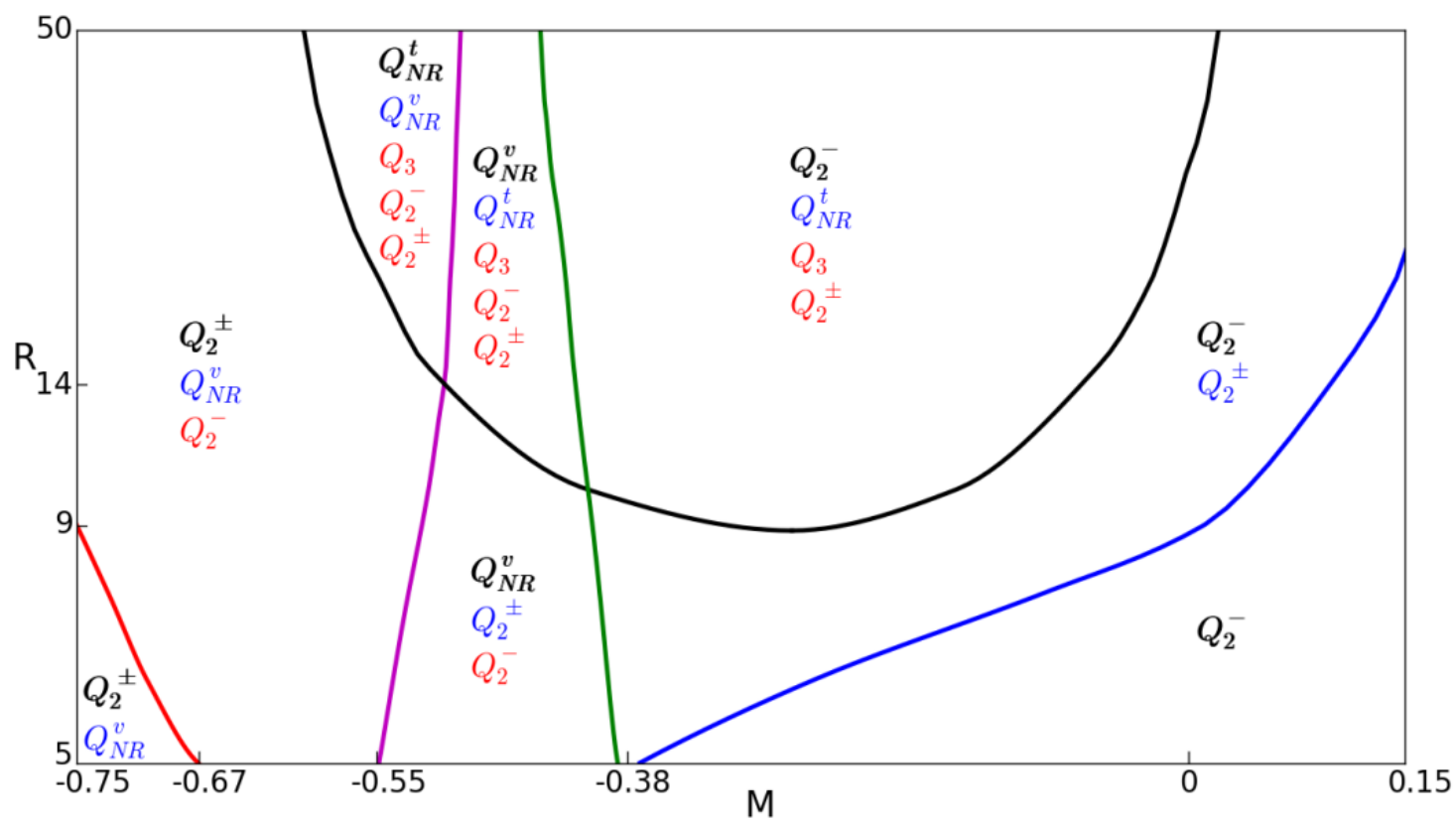

Figure 1: Log-log plot of the phase diagram for $b=0$ as a function of elastic constant $M$ and disk radius $R$ scaled as indicated, with $a=c=L=1$. Global minimisers of the Landau-de Gennes energy (1.11) are indicated in black; local minimisers, in blue; saddle points that are local minimisers of the reduced energy (2.8) or the 2-component restriction (2.13), in red.

Even with a partial set of critical points, we can discern a rich bifurcation set. The black curve in Figure 1 marks a pitchfork bifurcation, where two three-component radial solutions $Q_{3}$ (distinguished by the sign of their $w_{3}$ components) emerge from the two-component solution $Q_{2}^{ \pm}$, which undergoes a change in stability. The fact that $Q_{2}^{-}$is the global minimiser and $Q_{2}^{ \pm}$and $Q_{3}$ saddle points in the region bounded by and above the black curve is consistent with analytical results for $M=0$ and small $L$ [11]. The black curve also marks the appearance of the tilted non-radial solution $Q_{N R}^{t}$ as a minimiser through a bifurcation with $Q_{2}^{ \pm}$. The blue curve demarcates the domain of existence of $Q_{2}^{ \pm}$as a minimiser of the restricted energy. The red curve marks the domain of existence of $Q_{2}^{-}$as a minimiser of the restricted energy. The magenta curve denotes a first-order phase transition between $Q_{2}^{ \pm}$and $Q_{N R}^{t}$ for small $R$ and between $Q_{N R}^{t}$ and $Q_{N R}^{v}$ for large $R$.

Next, we examine the profiles of the critical points identified in Figure 1. The radiallysymmetric solutions $Q_{2}^{-}, Q_{2}^{ \pm}$and $Q_{3}$ are shown in Figure 3, including their radial components and $Q$-tensor plots.

We see that the radial components $w_{0}$ and $w_{1}$ of $Q_{2}^{-}$are monotonic, with $w_{0}<0$ and $w_{1}>0$. In contrast, for $Q_{2}^{ \pm}, w_{0}$ changes sign and $w_{1}$ is not monotonic. From the $Q$-tensor 

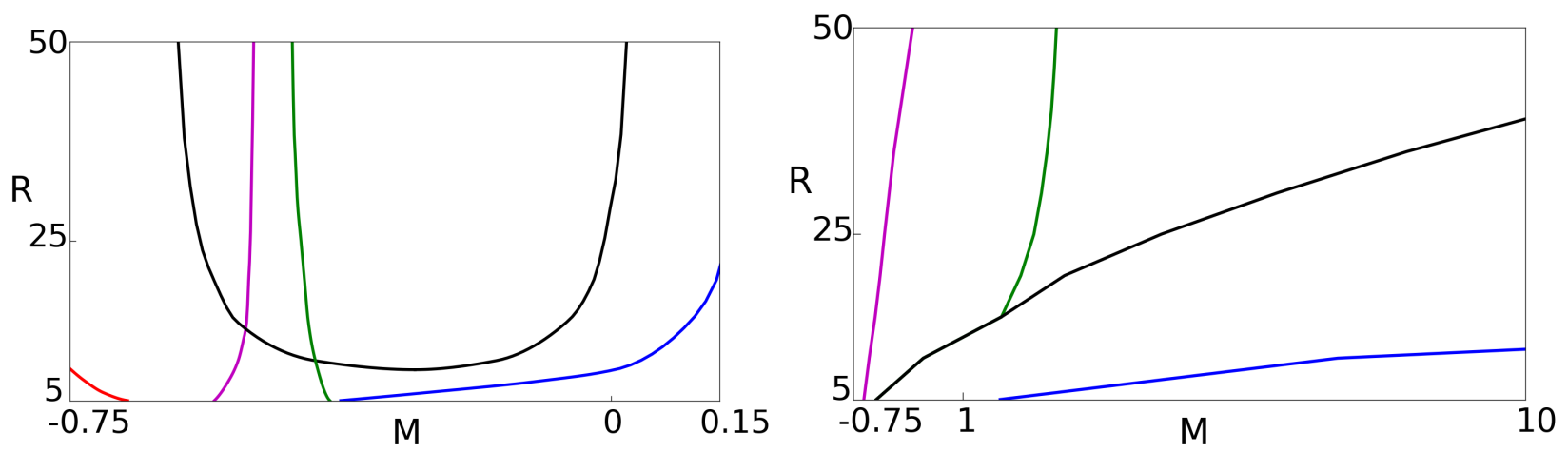

Figure 2: Unscaled bifurcation diagrams corresponding to Figure 1 (left) and Figure 5 (right).

plots, it is apparent that as the origin is approached, $Q_{2}^{-}$becomes oblate nematic and $Q_{2}^{ \pm}$ prolate nematic, with the director along $e_{3}$ for both. As noted above, $Q_{3}$ bifurcates from $Q_{2}^{ \pm}$ with $w_{3}$ becoming either positive or negative (the $Q_{3}$ profile shown in Figure 3 has $w_{3} \leq 0$ ). The qualitative similarity between the $w_{0}$ and $w_{1}$ components of $Q_{2}^{ \pm}$and $Q_{3}$ is apparent. The $Q$-tensor plot of $Q_{3}$ shows that the director (ie, the principal eigenvector of $Q_{3}$ ) rotates from $e_{3}$ at the origin to $n$ at the boundary, reminiscent of the escape-to-the-third-dimension (or skyrmion) solution in Oseen-Frank theory [8].

The plots shown in Figure 3 are for $M=0$. In this case, explicit formulas for the corresponding solutions are provided in the limit $L \rightarrow 0$ by Theorem 4.6 of [11], where, in particular, $Q_{3}$ becomes everywhere uniaxial, and the properties described above may be derived from these limiting formulas. For $M \neq 0$, the qualitative behaviour of these radially-symmetric solutions is found to be the same.

The non-radially symmetric solutions $Q_{N R}^{v}$ and $Q_{N R}^{t}$ are shown in Figure 4 with $M=$ -0.5 and $R=50$. We see from Figure 1 that $Q_{N R}^{v}$ is the global minimiser and $Q_{N R}^{t}$ is a local minimiser in this regime. In addition to $Q$-tensor fields, we plot the biaxiality parameter, $\beta$, given by

$$
\beta=1-6 \frac{\left(\operatorname{tr}\left(Q^{3}\right)\right)^{2}}{\left(\operatorname{tr}\left(Q^{2}\right)\right)^{3}}
$$

It is straightforward to show that $\beta$ takes values between 0 and 1 , and that $\beta=0$ if and only if $Q$ is uniaxial, while $\beta=1$ if and only if $Q$ is maximally biaxial, i.e. the eigenvalues of $Q$ are equally spaced (equivalently, $\operatorname{det} Q=0$ ). The biaxiality plots reveal two half-defects in $Q_{N R}^{v}$ and $Q_{N R}^{t}$ equally spaced about the origin on the $e_{1}$-axis, where $\beta=0$. As discussed above, $Q_{N R}^{v}$ has four discrete symmetries and $Q_{N R}^{t}$ only two. In particular, $Q_{N R}^{v}$ has $e_{3}$ as an eigenvector at the origin, while $Q_{N R}^{t}$ does not, as is apparent in Figure 4 . From the biaxiality plots, it is apparent that neither solution is uniaxial along the $y$-axis, but $Q_{N R}^{v}$ is much closer to being uniaxial there than is $Q_{N R}^{t}$. 

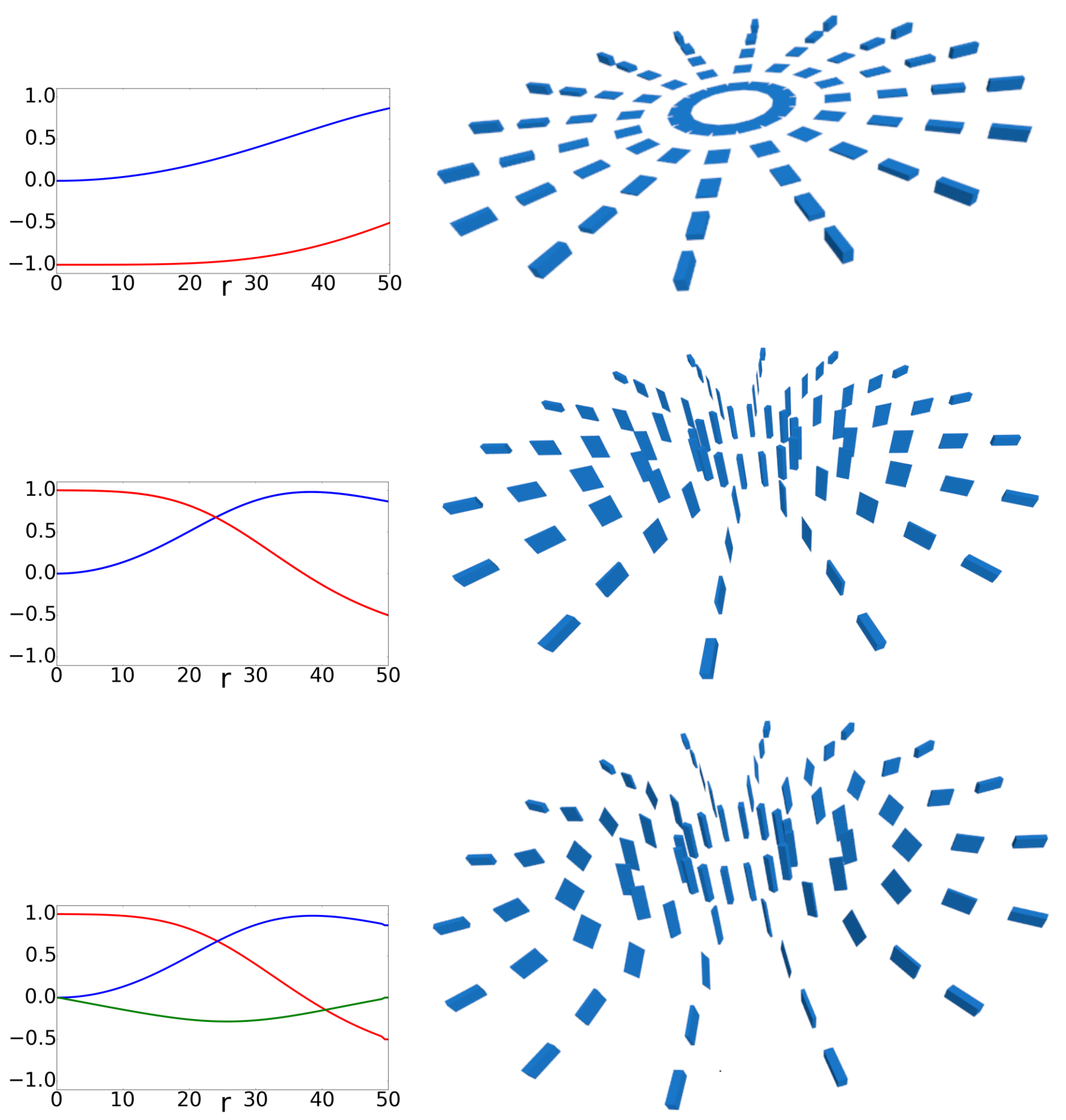

Figure 3: Radial components (left) and Q-tensor fields (right) for $Q_{2}^{-}$(top), $Q_{2}^{ \pm}$(middle), and $Q_{3}$ (bottom) for $M=0$ and $R=50$. As above, $b=0$ and $a=c=L=1$. In the radial-component plots on the left, $w_{0}$ is indicated in red, $w_{1}$ in blue, and $w_{3}$ in green. In the $Q$-tensor plots on the right, $Q$-tensors are represented as parallelepipeds with axes parallel to the eigenvectors of $Q(x)$ and with (nonnegative) lengths given by the eigenvalues of $Q(x)$ augmented by adding $\sqrt{\frac{2}{3}}|Q(x)|$ (see [33]). 

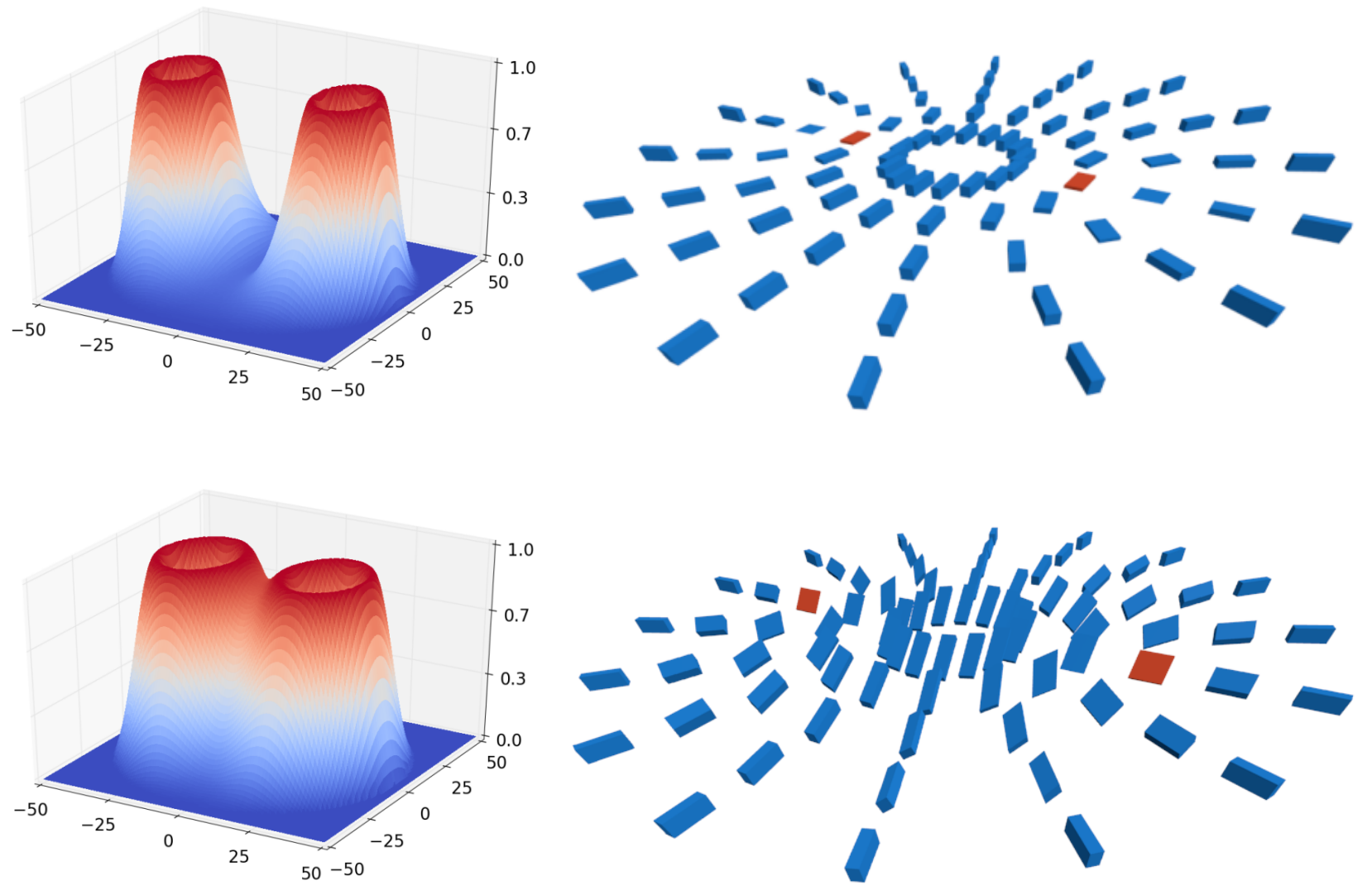

Figure 4: $\beta$ contours (left) and $Q$-tensor fields (right) for $Q_{N R}^{v}$ (top) and $Q_{N R}^{t}$ (bottom) for $M=-0.5$ and $R=50$. As above, $b=0$, and $a=c=L=1$ throughout. The location of the defects is indicated by the red boxes in the $Q$-tensor plots.

\subsection{Case $b=1$}

Following the numerical procedure described above, we obtain in Figure 5 a partial phase diagram for the Landau-de Gennes energy with $b=1$. Global minimisers, local minimisers and saddle points are as indicated. We find several differences in the set of solutions in comparison to the $b=0$ case. Among radially symmetric solutions, we find a single twocomponent solution $Q_{2}$, which is defined everywhere above the coercivity threshold and which interpolates between the characteristic properties of $Q_{2}^{-}$and $Q_{2}^{ \pm}$as $M$ decreases. We also find a five-component radial solution $Q_{5}$, which was not found for $b=0$. Among non-radially symmetric solutions, we find only $Q_{N R}^{v}$.

In the left-most region of phase plane (large negative $M$ ), $Q_{3}$ is the global minimiser. In the right-most region of the phase plane (sufficiently positive $M$ ), $Q_{2}$ is the global minimiser. In the region of intermediate $M$, the non-radially symmetric solution $Q_{N R}^{v}$ is the global minimiser. For $M=0$, these results are consistent with those of [17]. 
Even with a partial set of critical points, we find a rich bifurcation set. The magenta curve describes a first-order phase transition between $Q_{3}$ and $Q_{N R}^{v}$. The green curve describes a bifurcation in which $Q_{5}$ emerges from $Q_{3}$ (note that $Q_{3}$ is not indicated on the right side of the curve, as it ceases to be a local minimiser of the reduced energy (2.8) there). The black curve describes a bifurcation in which $Q_{5}$ and $Q_{3}$ change stability for large and small radii respectively. The blue curve describes a bifurcation in which $Q_{2}$ changes stability and $Q_{N R}^{v}$ emerges from it through a transcritical bifurcation.

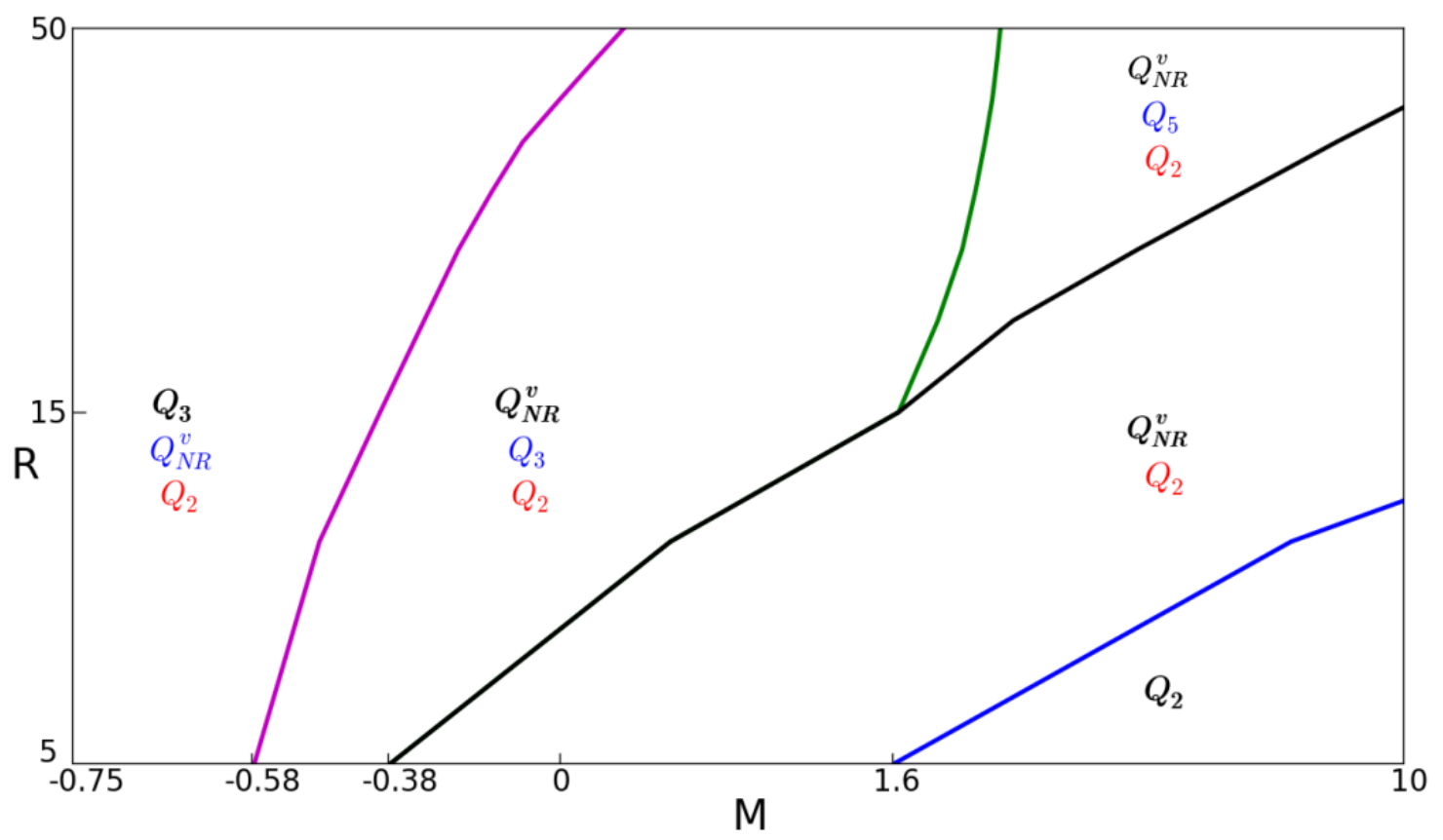

Figure 5: Log-log plot of existence and stability diagram in case $b=1$.

Next, we examine the profiles of the critical points identified in Figure 5. In Figure 6 we plot the profiles and $Q$-tensor fields for the two-component solution $Q_{2}$ for three characteristic values of $M$ : very large positive $M$, moderate positive $M$, and negative $M$. In contrast to the case $b=0$, as $M$ varies and $R$ is kept fixed, the $w_{0}$ component of the profile changes continuously from being strictly negative to sign-changing. As shown in Lemma 2.2, for large $M, w_{0}$ and $w_{1}$ are related by the first-order differential equation (2.16); for the first profile in Figure 6 with $M=100$, this is verified within numerical accuracy. 

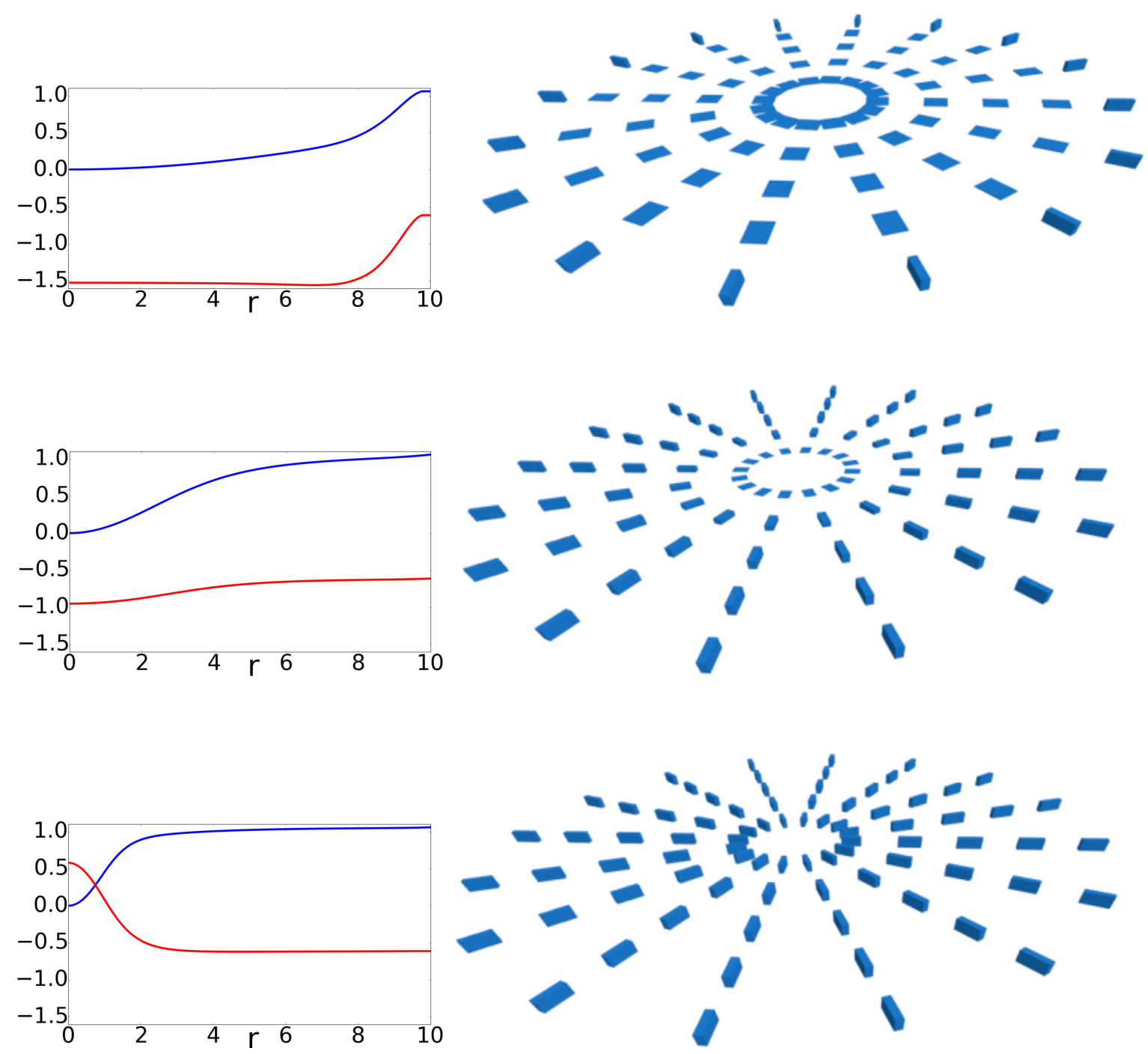

Figure 6: Radial components (left) and Q-tensor fields (right) of $Q_{2}$ for $R=10$ and $M=100$ (top row), where $Q_{2}$ is the global minimiser, and $M=1$ (middle row) and $M=-0.55$ (bottom row), where $Q_{2}$ is a saddle point. As above, $b=1$ and $a=c=L=1$ throughout. In the radialcomponent plots on the left, $w_{0}$ is indicated in red and $w_{1}$ in blue. In the $Q$-tensor plots on the right, $Q$-tensors are represented as parallelepipeds with axes parallel to the eigenvectors of $Q(x)$ and with (nonnegative) lengths given by the eigenvalues of $Q(x)$ augmented by adding $\sqrt{\frac{2}{3}}|Q(x)|$.

The radially symmetric solutions $Q_{3}$ and $Q_{5}$ are shown in Figure 7 for $R=50$ and different values of $M$. Recalling that $Q_{5}$ bifurcates from $Q_{3}$, we observe that their radial components $w_{0}, w_{1}$ and $w_{3}$ are similar. The $Q$-tensor plots show that $Q_{3}$ has the structure of the escape-to-the-third-dimension profile [8], while $Q_{5}$ is a twisted version of this profile. 

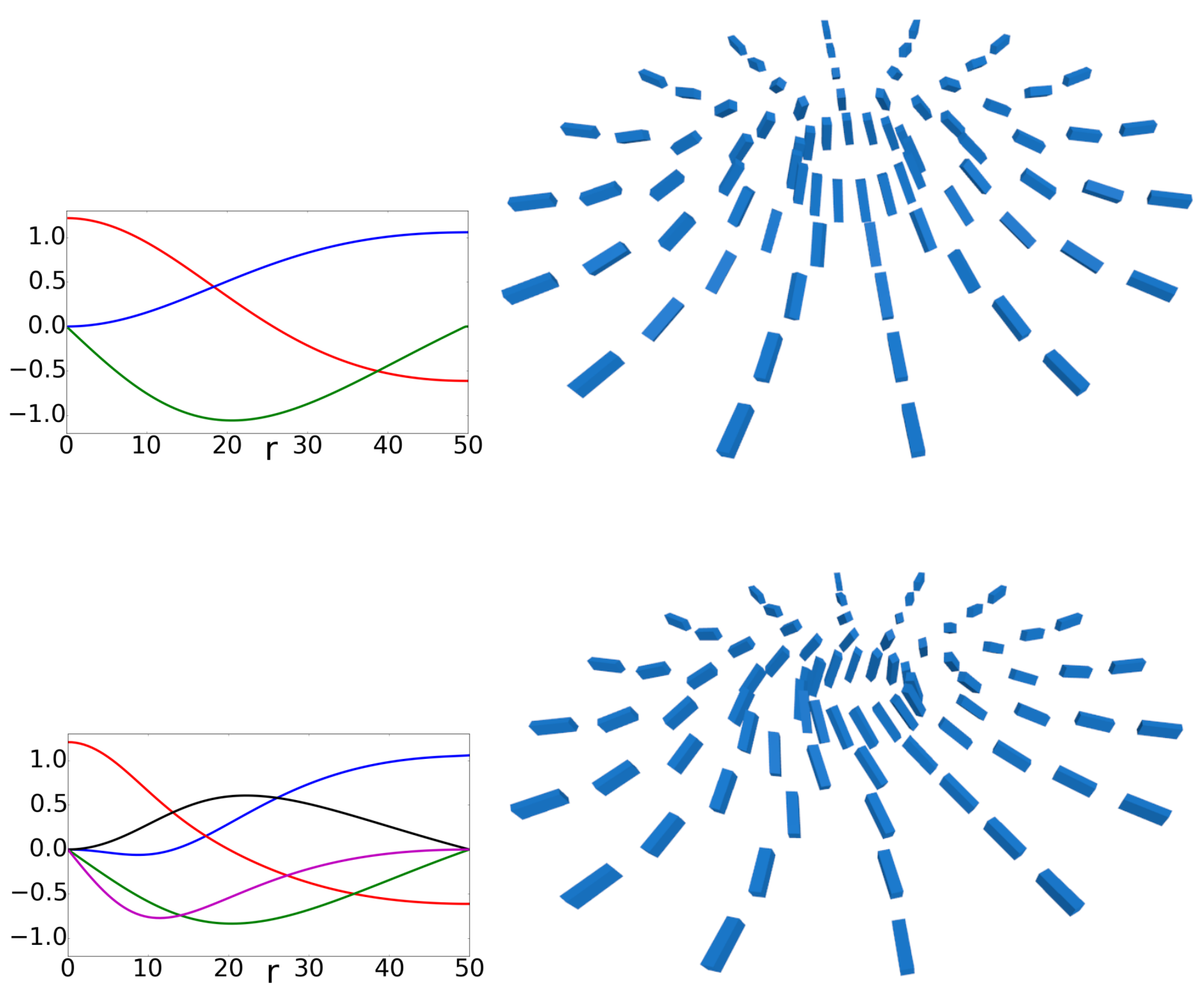

Figure 7: Radial profiles (left) and Q-tensor fields (right) for $R=50$ for $Q_{3}$ (top row) with $M=0$, where $Q_{3}$ is the global minimiser, and $Q_{5}$ (bottom row) with $M=5$, where $Q_{5}$ is a local minimiser. As above, $b=1$, and $a=c=L=1$ throughout. In the radial-component plots on the left, $w_{0}$ is indicated in red, $w_{1}$ in blue, $w_{2}$ in black, $w_{3}$ in green and $w_{4}$ in magenta.

The non-radially symmetric solution $Q_{N R}^{v}$ is shown in Figure 8 for $R=10$ and two values of $M$, namely $M=0$ and $M=5$. From the $\beta$-contour plots, it is apparent that region of biaxiality is concentrated around the two defects for $M=0$, while for $M=5$ it extends to a neighbourhood of the line joining the defects. 

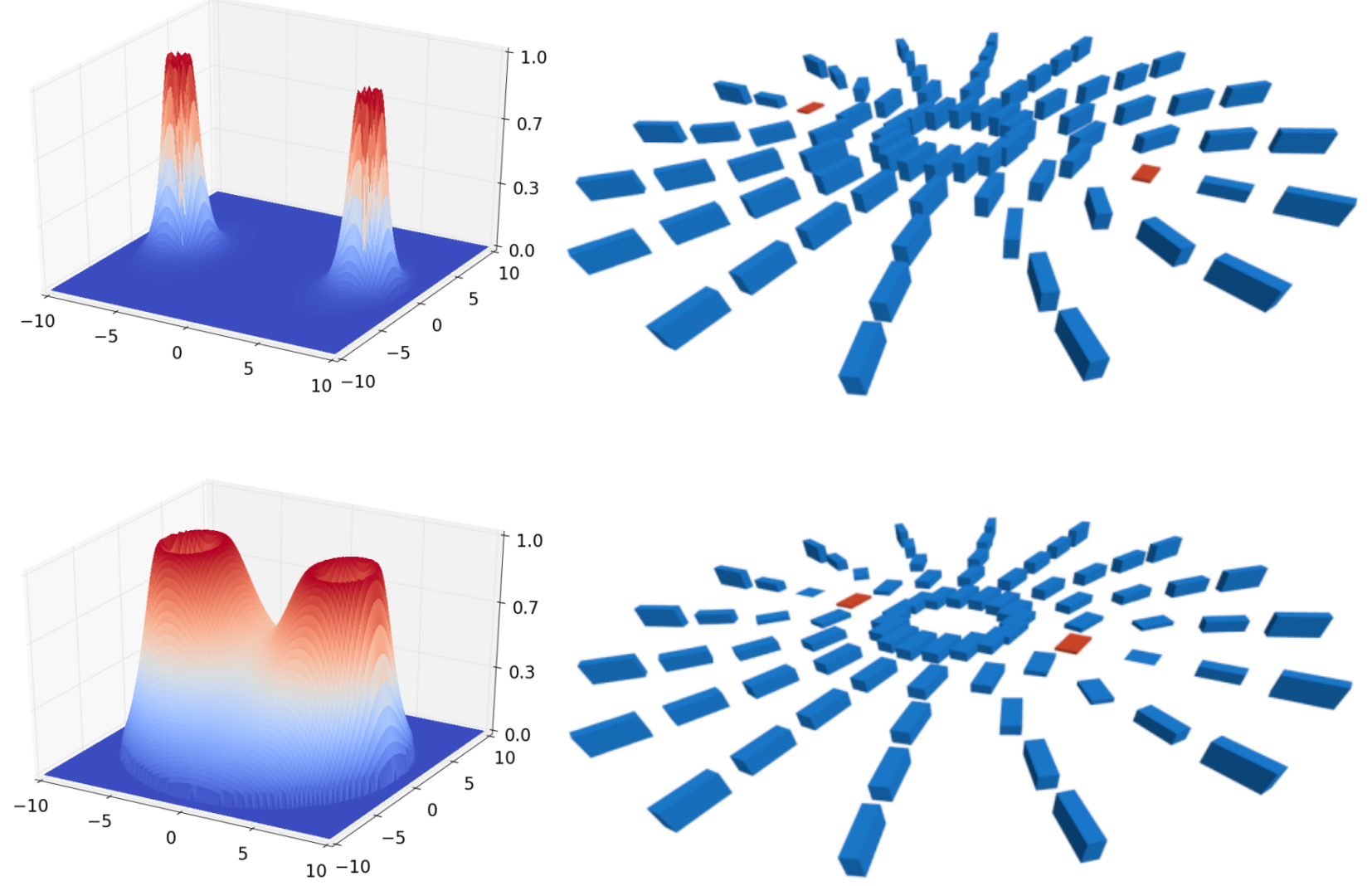

Figure 8: $\beta$ contours (left) and $Q$-tensor fields (right) for $Q_{N R}^{v}$ with $R=50$ and for $\mathrm{M}=0$ (first row) and $\mathrm{M}=5$ (second row). As above, $b=1$, and $a=c=L=1$. The location of the defects is indicated by the red boxes in the $Q$-tensor plots.

\section{Symmetry breaking of radially symmetric solutions}

As shown in Theorem 1.7, for $k \neq 2$ there are no generally radially symmetric critical points of the Landau-de Gennes energy when $M \neq 0$. We can employ formal perturbation theory to investigate how radial symmetry is broken for $M$ nonzero but small. We restrict our attention to the physically interesting cases $k= \pm 1$. Here, for $M=0$, the radial solutions are known to be local minimisers for $b>0$ (see [22]) and global minimisers for $b=0$ (see $[11])$.

Taking $M=\epsilon$, we write the Euler-Lagrange equation in the form

$$
L \Delta Q+a^{2} Q+b^{2}\left(Q^{2}-\frac{1}{3}|Q|^{2} I\right)-c^{2} Q|Q|^{2}=-\epsilon \mathcal{L} Q
$$

where $\mathcal{L} Q$ is defined in $(2.2)$. Let $Y=w_{0}(r) E_{0}+w_{1}(r) E_{1}$, with $w_{0}<0$ and $w_{1}>0$, denote 
the radially symmetric solution for the one-constant (i.e., $M=0$ ) Landau-de Gennes energy that is a local minimiser for $b>0$ [11] and a global minimiser for $b=0$ [11]. Writing $Q=Y+\epsilon W$, substituting above and ignoring terms of order $\epsilon^{2}$ and higher, we get the following linear inhomogeneous equation for $W$ :

$$
L \Delta W+a^{2} W+b^{2}\left(Y W+W Y-\frac{2}{3} \operatorname{tr}(Y W) I\right)-c^{2} W\left|Y^{2}\right|-2 c^{2} Y \operatorname{tr}(Y W)=-\mathcal{L} Y .
$$

The operator on the left-hand side of (4.2) corresponds to the second variation of the oneconstant Landau-de Gennes energy evaluated at the radially symmetric solution $Y$. For $0<b \leq \frac{75}{7} a^{2} c^{2}$, it has been shown that the second variation at $Y$ is strictly positive definite and it is conjectured that this holds for all $b>0[22]$. (For the special case $b=0, Y$ is the global minimiser, and the second variation is strictly positive for all $k$ [11]). Therefore, (4.2) has a unique solution. With some calculation one can show that the solution $W$ is of the form

$$
W=a_{0}(r) E_{0}+a_{1}(r) E_{1}+\cos ((k-2) \phi)\left(b_{0}(r) E_{0}+b_{1}(r) E_{1}\right)+\sin ((k-2) \phi) b_{2}(r) E_{2},
$$

where the five radial functions $a_{0}, a_{1}$ and $b_{0}, b_{1}, b_{2}$ satisfy a system of linear inhomogeneous ODE's, whose explicit expression has been omitted for brevity. It is clear that $a_{0}$ and $a_{1}$ describe perturbations of $w_{0}$ and $w_{1}$ respectively, and therefore preserve radial symmetry, while the $b_{j}$ 's comprise the symmetry-breaking component of the perturbed solution, $W_{n r}$, given by

$$
W_{n r}=\cos ((k-2) \phi)\left(b_{0}(r) E_{0}+b_{1}(r) E_{1}\right)+\sin ((k-2) \phi) b_{2}(r) E_{2} .
$$

It is straightforward to solve the ODE system numerically to obtain the $a_{j}$ 's and $b_{j}$ 's. In Figure 9, we give $Q$-tensor plots for the unperturbed solution $Y$ and symmetry-breaking component $W_{n r}$ for $k= \pm 1$ and $b=0,1$. For $k=-1, W_{n r}$ three-fold symmetry, in accord with (4.4). The numerical computations confirm that the approximate solutions $Y+\epsilon W$ are close to the minimisers $Q_{* \epsilon}$ of the full energy, and that the norm difference $\Delta:=\left\|Q_{*}-Y-\epsilon W\right\|_{L^{2}}$ scales as $\epsilon^{2}$. For example, for the case $k=-1, M=\epsilon=0.1$ and $b=0$, we find that $\Delta /\|Y\|_{L^{2}}=0.024$. In principle, one can develop a formal perturbation expansion for the exact minimiser in the form $Q_{* \epsilon}=Y+\epsilon W+\epsilon^{2} W_{2}+\cdots$, and derive a linear inhomogeneous system of ODEs for $W_{n+1}$ in terms of $Y$ and $W, \ldots, W_{n}$.

The computations also reveal that the symmetry between $k$ and $-k$ is broken for $M \neq 0$. We note that for $M=0$, the functions $w_{0}(r)$ and $w_{1}(r)$ for $k=1$ and $k=-1$ are the same; the Euler-Lagrange equations depend only on $k^{2}$ (the $Q$-tensor configurations for $k=1$ and $k=-1$ are not the same, of course, as $E_{1}$ depends on the sign of $k$ ). For $M \neq 0$, the $k \rightarrow-k$ symmetry is broken, as is readily seen in Figure 9. This symmetry breaking in $k$ appears only in $W_{n r}$, as the $a_{0}$ and $a_{1}$ components depend only on $k^{2}$. 

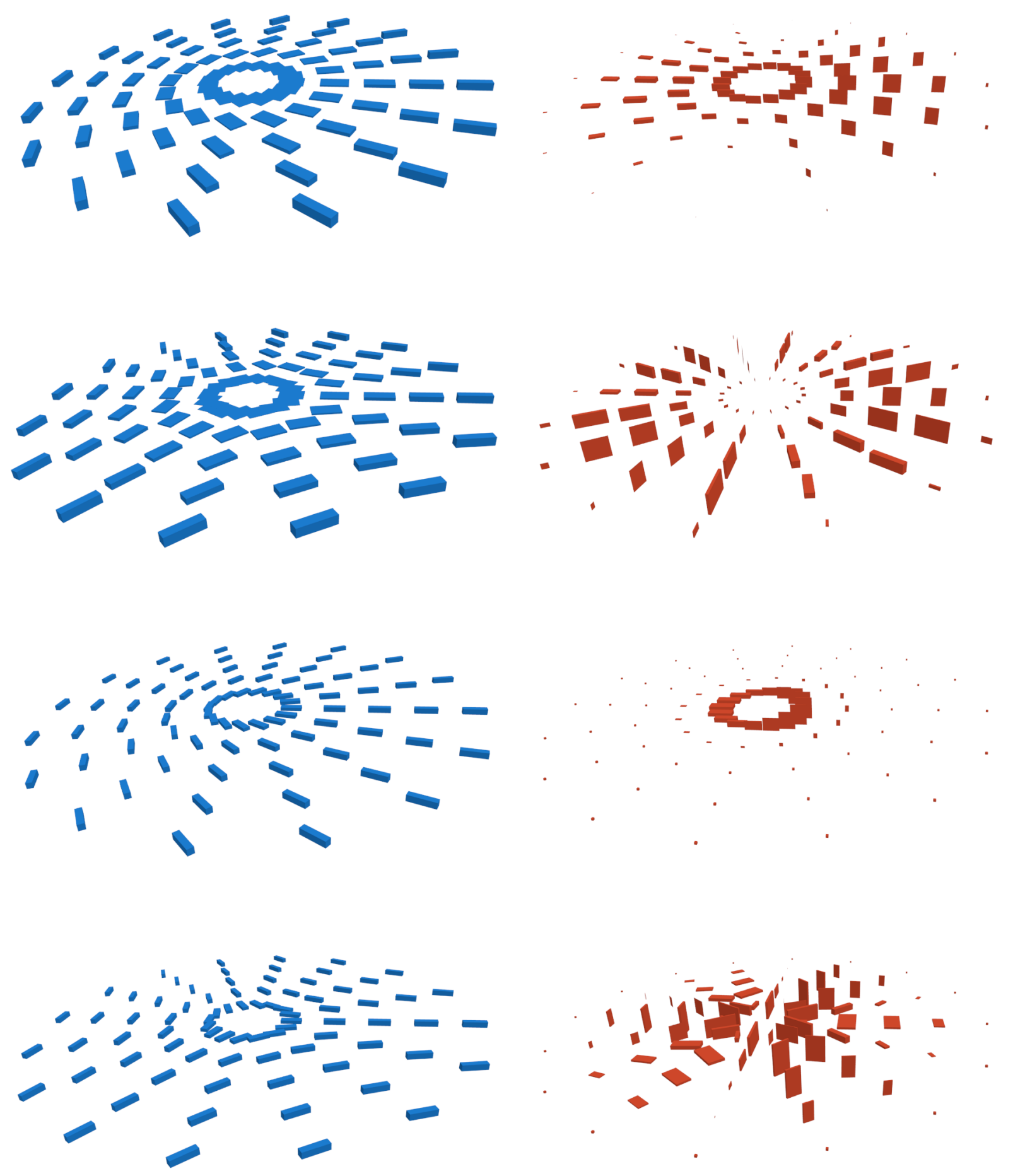

Figure 9: $Q$-tensor fields for the radially symmetric solutions (left) and the corresponding nonradial perturbations (4.4) (right) for $b=0, k=1, R=5$ (first row), $b=0, k=-1, R=5$ (second row), $b=1, k=1, R=2$ (third row), and $b=1, k=-1, R=2$ (fourth row). Throughout, $a=20$ and $L=c=1$. In the $Q$-tensor fields for the perturbations on the right, values of $W_{n r}$ very close to zero are indicated as points. 


\section{Conclusions and open problems}

The Landau-de Gennes theory with several elastic constants describes an extended range of rich behaviours in nematic liquid crystals as compared to the single-constant theory. Here we have considered several basic problems related to the existence and behaviour of generally radially symmetric critical points and their symmetry breaking. We have shown that generally radially symmetric critical points exist only in the case $k=2$, and we have investigated numerically the profiles and stability of these solutions. In particular, we have identified three types of radial profiles:

(a) two-component critical point $Q_{2}=w_{0}(r) E_{0}+w_{1}(r) E_{1}$;

(b) three-component critical point $Q_{3}=w_{0}(r) E_{0}+w_{1}(r) E_{1}+w_{3}(r) E_{3}$;

(c) five-component critical point $Q_{5}=\sum_{i=0}^{4} w_{i}(r) E_{i}$

and we have numerically investigated their minimality depending on the parameters $M, R$, and $b$ alongside non-symmetric solutions. Using the minimality property of two-component radial solutions in the case $M=0, k= \pm 1$ we have studied the symmetry breaking of these minimizers for small $M \neq 0$, identified directions of symmetry-breaking as solutions of a system of ODEs, and verified the principal results of this analysis through numerical simulations.

The model problem we have studied provides an excellent starting point for further analytical investigation of defects in the Landau-de Gennes theory. It is perhaps the simplest possible setting for developing the analytical tools necessary for attacking more general problems, particularly those in three-dimensional domains. Our investigations point to several natural open problems whose solution should lead to significant advances in the area.

\section{Profiles of radial solutions for $k=2$}

There are several important problems related to the qualitative features of the profiles of the radial solutions obtained in the Theorem 1.7. In the case $M=0$ the components $w_{0}$ and $w_{1}$ of 2-radial solution $Q_{2}^{-}$have constant sign and are monotone (see $[11,21,22]$ for analysis in 2D and [19] for analysis of a related 3D problem). This fact was particularly useful in the stability analysis when employing the Hardy decomposition trick (see [11, 21, 22]).

(a) Our numerical simulations indicate that for $M>0$ and $b>0$, the components $w_{0}$ and $w_{1}$ of the two-component solution $Q=w_{0}(r) E_{0}+w_{1}(r) E_{1}$ have definite sign. However, for $M<0$ we observe that $w_{0}$ changes sign. It is an interesting problem to explain this behaviour analytically.

(b) We observe numerically that for $M<0$ and small $b^{2}$, the radial components $w_{0}$ and $w_{1}$ of the two-component solution are no longer monotonic. It is an interesting problem to study the monotonicity of these radial profiles. Proving non-monotonicity is a challenging task, since there are no standard techniques available. This problem is also an opportunity 
to investigate the Landau-de Gennes system in a regime where it is no longer diagonal in the derivative terms.

(c) A challenging problem is to establish the existence and stability of the three-component and five-component radial solutions $Q_{3}$ and $Q_{5}$. We note that in some regimes (see Figures 3 and 7 ), the components of these solutions exhibit certain characteristics (eg, sign definiteness, monotonicity, convexity). Investigating these qualitative features may help to understand the stability properties of these solutions.

\section{Multiplicity of the local minimizers and critical points}

The problem of determining exactly, or even just lower and upper bounds for, the number of solutions for the nonlinear system of PDEs (or ODEs) is a very challenging and interesting problem. There exist several methods of nonlinear analysis, usually related to degree theory, that allow to show the existence of several critical points/local minimizers. However identifying all critical points (or even local minimizers) requires additional information, specific to the problem. The problems identified in this paper combine low dimensionality with the complexity of a coupled system of PDEs (or ODEs), and present an ideal playground for studying the question of multiplicity of solutions.

(a) Numerical minimization of the energy (2.13) indicates existence of at least three solutions in some range of parameters $M$ and $R$. Finding the lower and upper bounds on the number of critical points of this energy is the simplest possible problem related to the multiplicity of critical points of the full Landau-de Gennes energy. But even this problem presents a significant challenge due to nonlinear coupling in the Euler-Lagrange equations. We suggest to separately study cases $b=0$ and $b \neq 0$, as in the former case the equations are partially decoupled due to special form of nonlinearity.

(b) Finding lower and upper bounds on the number of critical points of the full radial energy (2.11) is a challenging mathematical problem. Numerical simulations indicate strong dependence of the number of critical points (and local minimizers) on $M, R$, and $b$.

(c) Identifying the exact number of critical point of the full Euler-Lagrange equations (1.16), (1.13) seems out of reach by current analytical methods. However, identifying lower and upper bounds on the number of symmetry-breaking solutions might be a first step towards the ultimate goal of classifying critical points of the Landau-de Gennes energy. For $M=0$, numerical simulations for $b>0$ indicate that there is at least one non-radial solution with $\mathbb{Z}_{k}$-symmetry [17].

\section{Bifurcations between various solutions}

Reconstruction of the bifurcation diagram is a question related to the multiplicity of solutions but involves more refined information about the system. Bifurcation problems are interesting and tractable because there are a number of powerful tools for studying them, see for instance [25]. A crucial step in studying various bifurcations in our model is the 
linearization of the Euler-Lagrange system near the known solution. The linear operator obtained in this way is essentially the quadratic form given by the second variation of the Landau-de Gennes energy. Thus, there exists a natural connection between bifurcation problems and the stability studies previously undertaken for these solutions [11, 21, 22]. Below are some bifurcation problems inspired by the numerical explorations reported here.

(a) There are three natural parameters, namely the radius $R$, the bulk parameter $b$ and the elastic constant $M$, and one can attempt to study bifurcations with respect to any one of these, holding the others fixed. One possible way to understand symmetry-breaking solutions is to use the fact near the bifurcation, the symmetry-breaking solution will be close to a radially symmetric solution, and the difference between them is related to the kernel of the linearisation at the radially symmetric solution (see for instance the standard bifurcation theorems of Crandall-Rabinowitz type in [25]).

(b) In the case $k=2$, there exist several types of radially symmetric solutions, depending on the value of $M$. A study of bifurcations of these radially symmetric solutions with $M$ as bifurcation parameter appears to be a simpler problem, but should provide important information about various types of critical points of the Landau-de Gennes energy.

\section{Global minimality}

Identifying a global minimizer is one of the most challenging problems in the calculus of variations. Most of the current methods allow for finding local minimizers, while proofs of global minimality are scarce and problem-dependent. Below we list several specific problems where we believe it may be possible to identify a global minimizer.

(a) It was shown in [11] that for $b=0$ and $M=0$, the 2-radially symmetric solution is the global minimizer of the Landau-de Gennes energy. It is an interesting question to determine to what extent this statement remains true in the case $M \neq 0$. We observe numerically that the two-component radial solution is the global minimizer for a range of $M$ and $R$ (see Figure 1). However, the qualitative features of the components (in particular being sign changing) depend on $M$ and $R$. Therefore it is not straightforward to adapt the proof in [11] for these regimes. A new proof of global minimality should probably depend on $M$ and $R$.

(b) In the case $M=0$ and $k= \pm 1$, the results in [22] show that the two-component radially symmetric solution $Q=w_{0}(r) E_{0}+w_{1}(r) E_{1}$ is a local minimizer. Our numerical simulations (see also [17]) suggest that this solutions is a global minimizer of Landau-de Gennes energy. It is an interesting and challenging problem to prove this fact analytically. Related but even more difficult is the question of uniqueness: are there any other local minimizers (or critical points) of the Landau-de Gennes energy (1.11) with boundary conditions (1.13) for $M=0$, $k= \pm 1$. 


\section{Acknowledgment.}

GK, JMR, VS would like to acknowledge support from Leverhulme grant RPG-2014-226. JMR and VS acknowledge support from EPSRC grant EP/K02390X/1. A.Z. was partially supported by a Grant of the Romanian National Authority for Scientific Research and Innovation, CNCS-UEFISCDI, project number PN-II-RU-TE-2014-4-0657; by the Basque Government through the BERC 2014-2017 program; and by the Spanish Ministry of Economy and Competitiveness MINECO: BCAM Severo Ochoa accreditation SEV-2013-0323. AZ gratefully acknowledges the hospitality of the Mathematics Department at the University of Bristol, through Leverhulme grant RPG-2014-226. VS and AZ gratefully acknowledge stimulating discussions with Prof. Radu Ignat and Prof. Luc Nguyen.

\section{Appendix}

\subsection{Null Lagrangian}

In this section we show that elastic terms with $L_{2}$ and $L_{3}$ are equivalent up to a boundary term. We prove the result in $\mathbb{R}^{3}$ and state the analogous result in $\mathbb{R}^{2}$.

Proof of Lemma 1.1. We assume $\Omega \subset \mathbb{R}^{3}$ with $C^{1}$ regular boundary $\partial \Omega$ and denote by $n$ the exterior unit normal to $\partial \Omega$. We define

$$
\mathcal{I}_{2} \stackrel{\text { def }}{=} \int_{\Omega} Q_{i k, j} Q_{i j, k}, \quad \mathcal{I}_{3} \stackrel{\text { def }}{=} \int_{\Omega} Q_{i j, j} Q_{i k, k}, \quad 1 \leq i, j, k \leq 3 .
$$

Straightforward calculation gives

$$
\begin{aligned}
\mathcal{I}_{2}-\mathcal{I}_{3} & =\int_{\Omega}\left(Q_{i j} Q_{i k, j}\right)_{, k}-\left(Q_{i j} Q_{i k, k}\right)_{, j} d x=\int_{\partial \Omega} Q_{i j} Q_{i k, j} n_{k}-Q_{i j} Q_{i k, k} n_{j} d \sigma \\
& =\int_{\partial \Omega} Q_{i j} Q_{i l, r}\left(\delta_{j r}-n_{j} n_{r}\right) n_{l}-Q_{i j} Q_{i k, l}\left(\delta_{k l}-n_{k} n_{l}\right) n_{j} d \sigma \\
& =\int_{\partial \Omega} W_{r j} T_{r j} d \sigma,
\end{aligned}
$$

where

$$
W_{r j}=n_{l}\left(Q_{, r} Q-Q Q_{, r}\right)_{l j}
$$

and $T:=I-n \times n$ is the orthogonal projection onto the tangent space of $\partial \Omega$. It is evident that $W_{r j} T_{r j}$ involves only $Q$ and its tangential derivatives on $\partial \Omega$, so that with prescribed Dirichlet boundary data $Q(x)=Q_{b}(x)$ for all $x \in \partial \Omega$, the value of $\mathcal{I}_{2}-\mathcal{I}_{3}$ is determined by $Q_{b}$. 
In order to obtain a relation between $\mathcal{I}_{2}$ and $\mathcal{I}_{3}$ in $\mathbb{R}^{2}$ we just assume that $Q$ is independent of variable $x_{3}$. Using (6.2) it is straightforward to obtain the analogous result for $\Omega \subset \mathbb{R}^{2}$,

$$
\mathcal{I}_{2}-\mathcal{I}_{3}=\int_{\partial \Omega} t_{j}\left(\frac{\partial Q_{i l}}{\partial t} Q_{i j}-\frac{\partial Q_{i j}}{\partial t} Q_{i l}\right) n_{l} d \sigma
$$

where $n=(\hat{n}, 0), t=(\hat{t}, 0)$, and $\hat{n}=\left(n_{1}, n_{2}\right), \hat{t}=\left(t_{1}, t_{2}\right)$ are normal and tangent to $\partial \Omega$, respectively.

\subsection{Coercivity conditions}

In this section we want to derive the coercivity conditions for the energy (1.11) in $\mathbb{R}^{3}$ and $\mathbb{R}^{2}$. The elastic part of the energy is

$$
\mathscr{F}_{e l}[Q ; \Omega]=\int_{\Omega}\left[\frac{L_{1}}{2}|\nabla Q|^{2}+\frac{L_{2}}{2} \partial_{j} Q_{i k} \partial_{k} Q_{i j}+\frac{L_{3}}{2} \partial_{j} Q_{i j} \partial_{k} Q_{i k}\right] d x .
$$

We prove Lemma 1.2 providing the necessary and sufficient relations between the elastic constants in order to have the coercivity conditions.

Proof of Lemma 1.2. In the following we argue for smooth $Q$-tensors, the reduction to the $H^{1}$ case being standard. As usual, we assume throughout summation over repeated indices.

1. We denote by $P_{i j k}, i, j, k=1,2,3$ the components of a third-order tensor

$$
P \in \mathscr{P}=\left\{P \in \mathbb{R}^{3 \times 3 \times 3}: P_{i j k}=P_{j i k}, P_{i i k}=0 \text { for all } 1 \leq i, j, k \leq 3\right\}
$$

and define the elastic energy term

$$
f(P):=L_{1} P_{i j k} P_{i j k}+L_{2} P_{i k j} P_{i j k}+L_{3} P_{i j j} P_{i k k} .
$$

In order to find relationships between $L_{1}, L_{2}$ and $L_{3}$ that ensure the coercivity we have to compute

$$
\min _{P \in \mathscr{P}, P_{i j k} P_{i j k}=1} f(P) .
$$

Note that we do not take into account the relation between $\mathcal{I}_{2}$ and $\mathcal{I}_{3}$ and we obtain pointwise constraints that give only sufficient conditions on the elastic constants $L_{i}$ to make the energy density in $\mathscr{F}_{\text {el }}$ positive definite pointwise.

In order to derive the coercivity conditions we define the Lagrangian function

$$
F(P, \lambda):=f(P)+\lambda\left(P_{i j k} P_{i j k}-1\right)+\mu_{j}\left(P_{l l j}\right)+\nu_{i j k}\left(P_{i j k}-P_{j i k}\right)
$$


whose critical points satisfy the equations

$$
\begin{gathered}
\frac{\partial F}{\partial P_{\alpha \beta \gamma}}=2\left(L_{1}+\lambda\right) P_{\alpha \beta \gamma}+2 L_{2} P_{\alpha \gamma \beta}+2 L_{3} P_{\alpha l l} \delta_{\beta \gamma}+\mu_{\gamma} \delta_{\alpha \beta}+\left(\nu_{\alpha \beta \gamma}-\nu_{\beta \alpha \gamma}\right)=0 \\
P_{\alpha \beta \gamma} P_{\alpha \beta \gamma}=1 \\
P_{\alpha \alpha \gamma}=0 \\
P_{\alpha \beta \gamma}=P_{\beta \alpha \gamma} .
\end{gathered}
$$

The last three equations are due to the constraints on $P$.

Multiplying (6.9) by $\delta_{\alpha \beta}$ and using (6.10), (6.11) and (6.12) we obtain

$$
\mu_{\gamma}=-\frac{2}{3}\left(L_{2}+L_{3}\right) P_{\gamma l l}
$$

Subtracting out of (6.9) the same equation but with $\alpha$ and $\beta$ interchanged, while using (6.12) we obtain

$$
\sigma_{\alpha \beta \gamma}:=\nu_{\alpha \beta \gamma}-\nu_{\beta \alpha \gamma}=L_{2}\left(P_{\beta \gamma \alpha}-P_{\alpha \gamma \beta}\right)+L_{3}\left(P_{\beta l l} \delta_{\alpha \gamma}-P_{\alpha l l} \delta_{\beta \gamma}\right)
$$

Multiplying (6.9) respectively by $P_{\alpha \beta \gamma}, P_{\alpha \gamma \beta}$ and using (6.10) we obtain the relations

$$
\begin{gathered}
2\left(L_{1}+\lambda\right)+2 L_{2} P_{\alpha \gamma \beta} P_{\alpha \beta \gamma}+2 L_{3}\left(P_{\alpha l l} P_{\alpha m m}\right)=0 \\
\left(L_{2}+2\left(L_{1}+\lambda\right)\right) P_{\alpha \gamma \beta} P_{\alpha \beta \gamma}+L_{2}+\left(\frac{L_{3}-2 L_{2}}{3}\right)\left(P_{\alpha l l} P_{\alpha m m}\right)=0 .
\end{gathered}
$$

Finally, taking $\beta=\gamma$ and multiplying (6.9) by $P_{\alpha l l}$, while taking into account that $\delta_{\gamma \gamma}=3$, we obtain

$$
\left(2\left(L_{1}+\lambda\right)+\frac{L_{2}}{3}+\frac{10}{3} L_{3}\right)\left(P_{\alpha \beta \beta} P_{\alpha l l}\right)=0 .
$$

In order to obtain the coercivity conditions on $L_{i}$ it suffices that the energy computed at any critical point is positive. We denote the elastic energy terms as

$$
H_{1}:=P_{\alpha \beta \gamma} P_{\alpha \gamma \beta}, \quad H_{2}:=P_{\alpha l l} P_{\alpha m m}
$$

and using equations (6.15)-(6.17) arrive at the following conditions:

- Case 1: $\mathrm{H}_{2}=0$. In this case out of (6.15) respectively (6.16) we get:

$$
H_{1}=-\frac{L_{1}+\lambda}{L_{2}}=-\frac{L_{2}}{L_{2}+2\left(L_{1}+\lambda\right)}
$$

and hence

$$
\frac{1}{H_{1}}=-\frac{L_{2}+2\left(L_{1}+\lambda\right)}{L_{2}}=-1+2 H_{1} .
$$


Solving the quadratic equation, we have

$$
H_{1} \in\left\{1,-\frac{1}{2}\right\}
$$

Using $H_{1}$ and $H_{2}$ in the energy we get the conditions:

$$
L_{1}+L_{2}>0, \quad 2 L_{1}-L_{2}>0 \text {. }
$$

- Case 2: $H_{2} \neq 0$. In this case

$$
2\left(L_{1}+\lambda\right)+\frac{L_{2}}{3}+\frac{10}{3} L_{3}=0
$$

and we can find

$$
L_{1}+\lambda=-\frac{L_{2}}{6}-\frac{5}{3} L_{3} .
$$

Plugging it into (6.15), (6.16) we obtain the following system

$$
\begin{gathered}
L_{2} H_{1}+L_{3} H_{2}=\frac{L_{2}}{6}+\frac{5}{3} L_{3}, \\
\left(\frac{2 L_{2}}{3}-\frac{10}{3} L_{3}\right) H_{1}+\left(\frac{L_{3}}{3}-\frac{2 L_{2}}{3}\right) H_{2}=-L_{2} .
\end{gathered}
$$

Since the energy is $f(P)=L_{1}+L_{2} H_{1}+L_{3} H_{2}$ using the first equation we immediately obtain

$$
L_{1}+\frac{L_{2}}{6}+\frac{5}{3} L_{3}>0
$$

and arrive at (1.6).

It straightforward to show that the coercivity conditions in $\mathbb{R}^{2}$ will be exactly as in (1.6).

2. Now we would like to see how coercivity conditions will be changed if we use Dirichlet boundary conditions and the equivalence of elastic constants $L_{2}$ and $L_{3}$, modulo boundary terms. We define

$$
M=\frac{L_{2}+L_{3}}{2}, L_{2}=\alpha M, L_{3}=(2-\alpha) M .
$$

Using the previous part we have:

$$
\int_{\Omega} L_{1} Q_{i j, k} Q_{i k, j}+L_{2} Q_{i k, j} Q_{i j, k}+L_{3} Q_{i j, j} Q_{i k, k} d x=\int_{\Omega} L_{1} Q_{i j, k} Q_{i j, k}+\underbrace{\left(L_{2}+L_{3}\right)}_{=2 M} Q_{i j, j} Q_{i k, k} d x
$$




$$
+L_{2} \int_{\Omega} Q_{i j, k} Q_{i k, j}-Q_{i j, j} Q_{i k, k} d x
$$

We note that because of Lemma 1.1 the last term is a constant, independent of $Q$, but depending just on $L_{2}$ and the boundary condition $Q=Q_{b}$ on $\partial \Omega$. Thus from the point of view of energy minimization we can ignore this constant and therefore the elastic constants $L_{2}$ and $L_{3}$ are interchangeable. We define the set $X_{\alpha}$

$$
X_{\alpha}=\left\{\left(L_{1}, M\right): L_{1}+\alpha M>0,2 L_{1}-\alpha M>0, L_{1}+\frac{\alpha}{6} M+\frac{5(2-\alpha)}{3} M>0\right\}
$$

and note that for each $\alpha$ the set $X_{\alpha}$ defines the corresponding coercivity conditions. Since $\alpha$ can be arbitrary the new coercivity conditions set $X$ is defined as $X=\cup_{\alpha} X_{\alpha}$. It is straightforward to obtain that

$$
X=\left\{\left(L_{1}, M\right): L_{1}+\frac{4}{3} M>0, L_{1}>0\right\} .
$$

Similar considerations hold for $2 D$ domains.

\subsection{Relation between $2 \mathrm{D}$ and $3 \mathrm{D}$ solutions}

In this section we want to investigate the relation between the critical points of the Landaude Gennes energy in a 2D domain $\omega$ and the corresponding critical points of LDG energy in the 3D cylinder $\Omega=\omega \times(-d, d)$. It is well known that when $L_{2}=L_{3}=0$ the critical point of 2D Landau-de Gennes energy is in fact a translation invariant critical point of the 3D Landau-de Gennes energy. Here we show that this is not necessarily the case when $L_{2}+L_{3} \neq 0$.

Critical points of the 2D Landau-de Gennes energy (1.11) with Dirichlet boundary conditions

$$
Q(x)=Q_{0}(x) \text { for } x \in \partial \omega
$$

satisfy the following weak form of the Euler-Lagrange equations:

$$
\int_{\omega}\left[L_{1} \partial_{k} Q_{i j} \partial_{k} R_{i j}+L_{2} \partial_{j} Q_{i k} \partial_{k} R_{i j}+L_{3} \partial_{k} Q_{i k} \partial_{j} R_{i j}+\partial f_{B}\left(Q_{i j}\right) R_{i j}\right] d x=0,
$$

where $R(x) \in \mathscr{S}_{0}$ for any $x \in \omega$ and $R(x)=0$ for $x \in \partial \omega$. The summation is taken over $i, j, k=1,2,3$, and since we work in a two-dimensional domain, we assume $Q_{i j, 3}=R_{i j, 3} \equiv 0$.

Taking $z$-invariant boundary conditions in 3D,

$$
Q(x, z)=Q_{0}(x) \text { for }(x, z) \in \partial \omega \times(-d, d)
$$


and computing the first variation of the Landau-de Gennes energy we obtain

$$
\int_{\Omega}\left[L_{1} \partial_{k} Q_{i j} \partial_{k} P_{i j}+L_{2} \partial_{j} Q_{i k} \partial_{k} P_{i j}+L_{3} \partial_{k} Q_{i k} \partial_{j} P_{i j}+\partial f_{B}\left(Q_{i j}\right) P_{i j}\right] d x=0,
$$

where $P(x, z) \in \mathscr{S}_{0}$ for any $(x, z) \in \Omega$ and $P(x, z)=0$ for any $(x, z) \in \partial \omega \times(-d, d)$.

We would like to show that unlike in the case $L_{2}+L_{3}=0$ the minimizer in $2 \mathrm{D}$ will not necessarily satisfy the Euler-Lagrange equations for the $3 \mathrm{D}$ problem. It is not difficult to see that (6.20) produces "natural boundary conditions" at the top and bottom of the cylinder,

$$
\int_{\omega} L_{1} \partial_{3} Q_{i j}(x, \pm d) P_{i j}(x, \pm d)+L_{2} \partial_{j} Q_{i 3}(x, \pm d) P_{i j}(x, \pm d)+L_{3} \partial_{k} Q_{i k}(x, \pm d) P_{i 3}(x, \pm d) d x=0 .
$$

Assuming that the 3D minimizer $Q_{i j}$ is independent of $z$, we immediately obtain

$$
L_{2}\left(\partial_{j} Q_{i 3}(x)+\partial_{i} Q_{j 3}(x)\right)+L_{3}\left(\partial_{k} Q_{i k}(x) \delta_{3 j}+\partial_{k} Q_{j k}(x) \delta_{3 i}\right)=0
$$

for all $x \in \omega$.

However, we also know that $Q$ has to satisfy Euler-Lagrange equations (1.16) with the boundary data $Q(x)=Q_{0}(x)$ for $x \in \partial \omega$. Therefore the nonlinear second order PDE has to be consistent with the linear first order PDE in (6.21), and in general this is not true. For example, it can be shown that the two-dimensional radially symmetric solutions we have considered here do not extend to $z$-independent solutions in three dimensions if $L_{2}+L_{3} \neq 0$.

\subsection{Symmetry breaking}

In this section we compute the symmetry breaking term

$$
\mathcal{L} Q_{i j}=\partial_{j} \partial_{k} Q_{i k}+\partial_{i} \partial_{k} Q_{j k}-\frac{2}{3} \partial_{l} \partial_{k} Q_{l k} \delta_{i j}
$$

where $Q$ is defined as

$$
Q=v(r) E_{0}+u(r) E_{1}+w(r) E_{2}
$$

It is straightforward to compute

$$
\mathcal{L}\left(v E_{0}\right)=-\frac{2}{\sqrt{6}}\left(\{\text { Hessf }\}-\frac{1}{3} \Delta v I_{3}\right)
$$

where $f(x)=v(|x|)$. Now we want to find $\mathcal{L}\left(u E_{1}\right)$ and $\mathcal{L}\left(u E_{2}\right)$. We first compute the generic term (abusing notation we say $E^{1}=E_{1}, E^{2}=E_{2}$ ),

$$
\partial_{j} \partial_{m}\left(u(r) E_{i m}^{1}\right)=\left(\partial_{j} \partial_{m} u(r)\right) E_{i m}^{1}+\left(\partial_{m} u(r)\right) \partial_{j} E_{i m}^{1}+\left(\partial_{j} u(r)\right) \partial_{m} E_{i m}^{1}+u(r) \partial_{j} \partial_{m} E_{i m}^{1} .
$$


It is straightforward to obtain the following relations:

$$
\begin{gathered}
\partial_{j} u(r)=u^{\prime}(r) \frac{x_{j}}{r}, \quad \partial_{j} \partial_{m} u(r)=\left(u^{\prime \prime}-\frac{u^{\prime}}{r}\right) \frac{x_{j} x_{m}}{r^{2}}+\frac{u^{\prime}}{r} \delta_{j m} ; \quad j, m=1,2 \\
\partial_{j} E^{1}=\frac{k x_{j}^{\perp}}{\sqrt{2} r^{2}}\left(\begin{array}{cc}
-\sin (k \varphi) & \cos (k \varphi) \\
\cos (k \varphi) & \sin (k \varphi)
\end{array}\right), \quad \partial_{m} E \cdot m=\frac{k}{\sqrt{2} r}\left(\begin{array}{c}
\cos ((k-1) \varphi) \\
\sin ((k-1) \varphi)
\end{array}\right),
\end{gathered}
$$

where we use notation $x^{\perp}=\left(-x_{2}, x_{1}, 0\right)$.

Using the above equalities we obtain

$$
\begin{aligned}
A_{11}=\partial_{1} \partial_{m}\left(u(r) E_{1 m}^{1}\right) & =\left(u^{\prime \prime}-\frac{u^{\prime}}{r}\right) \frac{x_{1}}{\sqrt{2} r} \cos ((k-1) \varphi)+\frac{u^{\prime}}{\sqrt{2} r} \cos (k \varphi) \\
& +\frac{k u^{\prime}}{\sqrt{2} r} \cos ((k-2) \varphi)+u \partial_{1}\left(\frac{k}{\sqrt{2} r} \cos ((k-1) \varphi)\right), \\
A_{12}=\partial_{2} \partial_{m}\left(u(r) E_{1 m}^{1}\right)= & \left(u^{\prime \prime}-\frac{u^{\prime}}{r}\right) \frac{x_{2}}{\sqrt{2} r} \cos ((k-1) \varphi)+\frac{u^{\prime}}{\sqrt{2} r} \sin (k \varphi) \\
& -\frac{k u^{\prime}}{\sqrt{2} r} \sin ((k-2) \varphi)+u \partial_{2}\left(\frac{k}{\sqrt{2} r} \cos ((k-1) \varphi)\right), \\
A_{21}=\partial_{1} \partial_{m}\left(u(r) E_{2 m}^{1}\right)= & \left(u^{\prime \prime}-\frac{u^{\prime}}{r}\right) \frac{x_{1}}{\sqrt{2} r} \sin ((k-1) \varphi)+\frac{u^{\prime}}{\sqrt{2} r} \sin (k \varphi) \\
+ & \frac{k u^{\prime}}{\sqrt{2} r} \sin ((k-2) \varphi)+u \partial_{1}\left(\frac{k}{\sqrt{2} r} \sin ((k-1) \varphi)\right), \\
A_{22}=\partial_{2} \partial_{m}\left(u(r) E_{2 m}^{1}\right)= & \left(u^{\prime \prime}-\frac{u^{\prime}}{r}\right) \frac{x_{2}}{\sqrt{2} r} \sin ((k-1) \varphi)-\frac{u^{\prime}}{\sqrt{2} r} \cos (k \varphi) \\
+ & \frac{k u^{\prime}}{\sqrt{2} r} \cos ((k-2) \varphi)+u \partial_{2}\left(\frac{k}{\sqrt{2} r} \sin ((k-1) \varphi)\right) .
\end{aligned}
$$

Now we are ready to find

$$
\mathcal{L}\left(u E_{1}\right)=\left(\begin{array}{ccc}
A_{11}-A_{22} & A_{12}+A_{21} & 0 \\
A_{12}+A_{21} & -A_{11}+A_{22} & 0 \\
0 & 0 & 0
\end{array}\right)+\frac{A_{11}+A_{22}}{3}\left(\begin{array}{ccc}
1 & 0 & 0 \\
0 & 1 & 0 \\
0 & 0 & -2
\end{array}\right) .
$$

Since

$$
E_{1 m}^{2}=-E_{2 m}^{1}, \quad E_{2 m}^{2}=E_{1 m}^{1}
$$


we can immediately obtain

$$
\mathcal{L}\left(u E_{2}\right)=\left(\begin{array}{ccc}
-A_{21}-A_{12} & -A_{22}+A_{11} & 0 \\
-A_{22}+A_{11} & A_{21}+A_{12} & 0 \\
0 & 0 & 0
\end{array}\right)+\frac{A_{12}-A_{21}}{3}\left(\begin{array}{ccc}
1 & 0 & 0 \\
0 & 1 & 0 \\
0 & 0 & -2
\end{array}\right) .
$$

It is not difficult to compute

$$
\begin{gathered}
A_{11}-A_{22}=\left(u^{\prime \prime}+\frac{u^{\prime}}{r}-\frac{k^{2} u}{r^{2}}\right) \frac{\cos (k \varphi)}{\sqrt{2}}, \\
A_{12}+A_{21}=\left(u^{\prime \prime}+\frac{u^{\prime}}{r}-\frac{k^{2} u}{r^{2}}\right) \frac{\sin (k \varphi)}{\sqrt{2}} \\
A_{11}+A_{22}=\left(u^{\prime \prime}+\frac{(2 k-1) u^{\prime}}{r}+\frac{k(k-2) u}{r^{2}}\right) \frac{\cos ((k-2) \varphi)}{\sqrt{2}}, \\
A_{12}-A_{21}=-\left(u^{\prime \prime}+\frac{(2 k-1) u^{\prime}}{r}+\frac{k(k-2) u}{r^{2}}\right) \frac{\sin ((k-2) \varphi)}{\sqrt{2}} .
\end{gathered}
$$

Combining the results above, we obtain

$$
\begin{gathered}
\mathcal{L}\left(v(r) E_{0}\right)=\frac{1}{3}\left(v^{\prime \prime}+\frac{v^{\prime}}{r}\right) E_{0}-\frac{1}{\sqrt{3}}\left(v^{\prime \prime}-\frac{v^{\prime}}{r}\right)\left(E_{1} \cos ((k-2) \varphi)-E_{2} \sin ((k-2) \varphi)\right) \\
\mathcal{L}\left(u(r) E_{1}\right)=\left(u^{\prime \prime}+\frac{u^{\prime}}{r}-\frac{k^{2} u}{r^{2}}\right) E_{1}-\frac{1}{\sqrt{3}}\left(u^{\prime \prime}+\frac{(2 k-1) u^{\prime}}{r}+\frac{k(k-2) u}{r^{2}}\right) \cos ((k-2) \varphi) E_{0}, \\
\mathcal{L}\left(w(r) E_{2}\right)=\left(w^{\prime \prime}+\frac{w^{\prime}}{r}-\frac{k^{2} w}{r^{2}}\right) E_{2}+\frac{1}{\sqrt{3}}\left(w^{\prime \prime}+\frac{(2 k-1) w^{\prime}}{r}+\frac{k(k-2) w}{r^{2}}\right) \sin ((k-2) \varphi) E_{0} .
\end{gathered}
$$

\section{References}

[1] BALL, J. M. Lecture notes on the $Q$-tensor theory of liquid crystals. Oxford University course, unpublished, 2006.

[2] Ball, J. M., And Majumdar, A. Nematic liquid crystals : from Maier-Saupe to a Continuum theory. Mol. Cryst. Liq. Cryst. 525 (2010), 1-11. 
[3] Ball, J. M., And Zarnescu, A. Orientability and energy minimization in liquid crystal models. Arch.Rational Mech. Anal 202, 2 (2011), 493-535.

[4] Bauman, P., And Philips, D. Analysis of nematic liquid crystals with disclination lines. Arch. Rational Mech. Anal 205 (2012), 795-826.

[5] Bauman, P., And Phillips, D. Regularity and the behavior of eigenvalues for minimizers of a constrained Q-tensor energy for liquid crystals. Calc. Var. Partial Differential Equations (2016), 55-81.

[6] Canevari, G. Biaxiality in the asymptotic analysis of a 2-d Landau-de Gennes model for liquid crystals. ESAIM: Control, Optimization and Calculus of Variations 21, 1 (2015), 101-137.

[7] Chandrasekhar, S., and Ranganath, G. The structure and energetics of defects in liquid crystals. Advances in Physics 35 (1986), 507-596.

[8] Cladis, P., And Kleman, M. Non-singular disclinations of strength $s=+1$ in nematics. J. Phys. 33 (1972), 591-598.

[9] Davis, T. A., and Gartland, E. C. Finite element analysis of the Landau-de Gennes minimization problem for liquid crystals. SIAM J. Numer. Anal. 35, 1 (1998), 336-362.

[10] De Gennes, P., And Prost, J. The physics of liquid crystals. Oxford University Press, Oxford, 1995. Second Edition.

[11] Di Fratta, G., Robbins, J., Slastikov, V., and Zarnescu, A. Half-integer point defects in the Q-tensor theory of nematic liquid crystals. Journal of Nonlinear Science 26, 1 (2016), 121-140.

[12] Ericksen, J. Inequalities in liquid crystal theory. Physics of Fluids (1958-1988) 9, 6 (1966), 1205-1207.

[13] Fatkullin, I., And Slastikov, V. On spatial variations of nematic ordering. Phys. D 237, 20 (2008), 2577-2586.

[14] Fatkullin, I., And Slastikov, V. Vortices in two-dimensional nematics. Commun. Math. Sci. 7, 4 (2009), 917-938.

[15] Gartland, E. C. Scalings and limits of the Landau-de Gennes model for liquid crystals: A comment on some recent analytical papers. arXiv preprint arXiv:1512.08164 (2015). 
[16] Golovaty, D., And Montero, A. On Minimizers of a Landau-de Gennes Energy Functional on Planar Domains. Arch. Rational Mech. Anal. 213 (2014), 447-490.

[17] Hu, Y., Qu, Y., And Zhang, P. On the disclination lines of nematic liquid crystals. Communications in Computational Physics 19 (2016), 354-379.

[18] Ignat, R., Nguyen, L., Slastikov, V., And Zarnescu, A. Stability of the vortex defect in the Landau-de Gennes theory for nematic liquid crystals. C. R. Math. Acad. Sci. Paris 351, 13-14 (2013), 533-537.

[19] Ignat, R., Nguyen, L., Slastikov, V., And Zarnescu, A. Uniqueness results for an ODE related to a generalized Ginzburg-Landau model for liquid crystals. SIAM J. Math. Anal. 46, 5 (2014), 3390-3425.

[20] Ignat, R., Nguyen, L., Slastikov, V., and Zarnescu, A. Stability of the melting hedgehog in the Landau-de Gennes theory of nematic liquid crystals. Arch. Ration. Mech. Anal. 215, 2 (2015), 633-673.

[21] Ignat, R., Nguyen, L., Slastikov, V., and Zarnescu, A. Instability of point defects in a two-dimensional nematic liquid crystal model. In Annales de l'Institut Henri Poincare (C) Non Linear Analysis (2016), vol. 33, Elsevier, pp. 1131-1152.

[22] Ignat, R., Nguyen, L., Slastikov, V., and Zarnescu, A. Stability of point defects of degree $\pm \frac{1}{2}$ in a two-dimensional nematic liquid crystal model. Calc.Var. Partial Differential Equations (2016).

[23] Iyer, G., Xu, X., And Zarnescu, A. D. Dynamic cubic instability in a 2d q-tensor model for liquid crystals. Mathematical Models and Methods in Applied Sciences 25, 08 (2015), 1477-1517.

[24] Katriel, J., Kventsel, G., Luckhurst, G., and Sluckin, T. Free energies in the landau and molecular field approaches. Liquid Crystals 1, 4 (1986), 337-355.

[25] KIELhÖFER, H. Bifurcation theory: An introduction with applications to PDEs, vol. 156. Springer Science \& Business Media, 2006.

[26] LAmy, X. Uniaxial symmetry in nematic liquid crystals. Annales de l'Institut Henri Poincare (C) Non Linear Analysis 32, 5 (2015), 1125-1144.

[27] Lin, F.-H., And Liu, C. Static and dynamic theories of liquid crystals. J. Partial Differential Equations 14, 4 (2001), 289-330.

[28] Longa, L., Monselesan, D., And Trebin, H. An extension of the LandauGinzburg-de Gennes theory for liquid crystals. Liq. Crys. 2 (1987), 769-796. 
[29] Majumdar, A., And Zarnescu, A. Landau-de Gennes theory of nematic liquid crystals: the Oseen-Frank limit and beyond. Arch. Ration. Mech. Anal. 196, 1 (2010), $227-280$.

[30] Mkaddem, S., and Gartland, E. C. Fine structure of defects in radial nematic droplets. Phys. Rev. E 62 (2000), 6694-6705.

[31] Mottram, N. J., And Newton, C. J. Introduction to Q-tensor theory. arXiv preprint arXiv:1409.3542 (2014).

[32] NGuyen, L., And Zarnescu, A. Refined approximation for minimizers of a landaude gennes energy functional. Calculus of Variations and Partial Differential Equations 47, 1-2 (2013), 383-432.

[33] Sonnet, A., Kilian, A., And Hess, S. Alignment tensor versus director: Description of defects in nematic liquid-crystals. Phys. Rev. E 52, 1 (1995), 718-722.

[34] VIRGA, E. G. Variational theories for liquid crystals, vol. 8 of Applied Mathematics and Mathematical Computation. Chapman \& Hall, London, 1994. 